\title{
Efficient choice of colored noise in the stochastic dynamics of open quantum systems
}

\author{
D. Matos $\odot,{ }^{1}$ M. A. Lane $\odot,{ }^{1}$ I. J. Ford, ${ }^{2}$ and L. Kantorovich ${ }^{1}$ \\ ${ }^{1}$ Department of Physics, King's College London, Strand, London WC2R 2LS, United Kingdom \\ ${ }^{2}$ Department of Physics and Astronomy, University College London, Gower Street, London WC1E 6BT, United Kingdom
}

(Received 2 June 2020; accepted 10 November 2020; published 16 December 2020)

\begin{abstract}
The stochastic Liouville-von Neumann (SLN) equation describes the dynamics of an open quantum system reduced density matrix coupled to a non-Markovian harmonic environment. The interaction with the environment is represented by complex colored noises which drive the system, and whose correlation functions are set by the properties of the environment. We present a number of schemes capable of generating colored noises of this kind that are built on a noise amplitude reduction procedure [Imai et al., Chem. Phys. 446, 134 (2015)], including two analytically optimized schemes. In doing so, we pay close attention to the properties of the correlation functions in Fourier space, which we derive in full. For some schemes the method of Wiener filtering for deconvolutions leads to the realization that weakening causality in one of the noise correlation functions improves numerical convergence considerably, allowing us to introduce a well-controlled method for doing so. We compare the ability of these schemes, along with an alternative optimized scheme [Schmitz and Stockburger, Eur. Phys. J.: Spec. Top. 227, 1929 (2019)], to reduce the growth in the mean and variance of the trace of the reduced density matrix, and their ability to extend the region in which the dynamics is stable and well converged for a range of temperatures. By numerically optimizing an additional noise scaling freedom, we identify the scheme which performs best for the parameters used, improving convergence by orders of magnitude and increasing the time accessible by simulation.
\end{abstract}

DOI: 10.1103/PhysRevE.102.062134

\section{INTRODUCTION}

In open quantum systems, interactions between the system of interest and its environment drive behaviors such as dissipation and decoherence which are not found in isolation. These play a strong role in quantum computing [1] where the ability of the open system to stay in a superposition of states is desirable and also in quantum thermodynamics [2]. Unfortunately, the very large number of environmental degrees of freedom makes the treatment of both the system and environment analytically and numerically challenging, especially when there is strong coupling between them. For this reason, existing methods tend to begin by taking the partial trace of the full density matrix over the environment variables to obtain the reduced density matrix of the system of interest. In particular, this is done in the well-known Feynman-Vernon influence functional formalism where the response of a bath coupled linearly to the open system is expressed as a path integral over an infinite number of displaced harmonic oscillators [3]. Techniques which build on this method include hierarchical equations of motion (HEOM) [4-6], hybrid stochastic HEOM [7,8], hierarchy of pure states [9], stochastic Schrödinger equations [10], quasiadiabatic path integrals [11], stochastic Liouvillevon Neumann equations (SLNs) [12-15], and the extended SLN (ESLN) equations method, which accounts for initial thermalization by the inclusion of an additional stochastic differential equation (SDE) in imaginary time with imaginary time noises [16-18]. Importantly, none of these methods make the Markov assumption, where the environment correlation times are taken to be negligibly short compared to the characteristic timescales of the open system. The Markov assumption has the physical interpretation that any information dissipated from the system to the environment will never be returned, i.e., the system-environment coupling is memoryless. Instead, the environment is allowed to be fully non-Markovian, introducing a source of memory to the system.

The SLN and ESLN methods, amongst others, are based on solving SDEs with complex correlated (colored) Gaussian noises. Beginning with the seminal work of Grabert, Schramm, and Ingold [19], these methods evolve stochastic reduced density matrices via SDEs, driven by the aforementioned noises, with the physical density matrix being recovered by stochastic averaging over all realizations of these noises. The advantage of these methods is that they are exact and nonperturbative and are in principle applicable to any temperature, system-environment coupling strength, and any form of the spectral density. In addition, with the recent development of the ESLN, the system and its environment can be thermalized via the application of an initial evolution in imaginary time, rather than being initialized in a partitioned state [12-15]. The current work focuses on the properties of the noises and their generation rather than on thermalization or the properties of specific physical dynamics, so we shall limit ourselves to the SLN rather than the ESLN for simplicity. It is important to note that these methods do not constitute an $a d$ hoc representation of the system behavior where the noises might have been introduced artificially to model the environment. Instead, they have been derived rigorously from 
an appropriate consideration of the whole system, consisting of both the open system and its environment, by means of elimination of the environment using the path-integral method and a Hubbard-Stratonovich transformation.

To simulate these SDEs, particular care must be taken when generating the complex colored noises, as the choice of a generation scheme can significantly alter the statistical properties of the noises and thus the system dynamics. A poor choice is characterized by a catastrophic loss of trace preservation for the reduced density matrix, which requires an exponentially large sample for convergence of the average. Making this choice is not trivial since the correlation functions must be satisfied with sensible decisions being made wherever there is freedom to do so, especially for stronger coupling when the magnitude of the noises is already large. In fact, one of the main conclusions of this work is that generating noises which satisfy the desired correlation functions is not enough to guarantee convergence or that the results be physical, despite the correlation functions being the only formal requirements of the theory on the noises.

In our previous work [18], one particular noise generation scheme was used which produced well-converged results as a verification of the ESLN method, but here we generalize our procedure and explore a number of possible noise generation schemes which all create the desired correlation functions but produce results of different convergence for the open system dynamics. We also optimize the scheme to minimize the (erroneous) exponential growth of the trace, something which has only recently been studied in any detail [18,20,21], though with some inconsistencies in [20] which we correct, and compare our optimized scheme with a recently proposed alternative, obtained independently via a different method and optimized subject to different constraints [21]. By examining the properties of the Fourier transforms of the desired correlations, the properties of the different noises, and their effect on the system dynamics, we arrive at a number of conclusions about noise generation for SLN methods, where and why issues arise, and how to maximize the possible duration (run time) of simulations before the stochastic nature of the dynamics inevitably leads to numerical blow up and statistical uncertainty.

For this purpose, we will use the spin-boson model as it is a relatively simple model consisting of a two-level spin system surrounded by bosonic degrees of freedom that describe the environment. This can naturally be applied to qubits coupled to an environment [22-26], electronic energy transfer in biological systems [20], Josephson junctions [27-29], cold atoms [30,31], and solid-state artificial atoms [32]. The spin-boson model has already been considered previously by us in the context of the ESLN [18].

Comparison with other methods mentioned in the Introduction is not within the scope of this paper, as it will be focusing only on the details of the noise generation within the SLN equation formalism. So, the purpose of the present paper is fourfold: (1) develop a general scheme for noise generation for the SLN equation and propose a number of possible choices for the scheme, including a fully optimized choice, (2) demonstrate that these choices significantly alter the properties of the noises with appropriate use of deconvolution methods [33,34] where necessary, (3) examine in detail how different choices affect the convergence properties of the results and the accessible run time of simulation before blow up, comparing with other optimization schemes where possible [20,21], and (4) explain in detail why particular choices fail, referring to the properties of the correlation functions themselves where necessary. More concretely, in Sec. II we briefly review the SLN formalism and the spin-boson model, before introducing in Sec. III our specific framework for noise generation and the possible choices we have identified. Finally in Sec. IV we present the results of the various noise generation schemes.

\section{THEORY}

\section{A. Stochastic Liouville-von Neumann equations}

Following the influence functional formalism of Feynman and Vernon [3], we consider the standard setup of an open quantum system with coordinates $q$ and Hamiltonian $H_{q}$ (that may describe either an electronic or bosonic subsystem, or both) coupled to an environmental heat bath of harmonic oscillators $i$ with masses $m_{i}$, governed by a potential energy that is quadratic in the oscillator displacement coordinates $\xi_{i}$. The coupling between the open system and its environment is linear in the environment coordinates but fully general in $q$, taking the form $-\xi_{i} f_{i}(q)$ for the given coordinate $\xi_{i}$, with the $f_{i}(q)$ being arbitrary functions of $q$. The full system Hamiltonian is thus

$$
\begin{aligned}
H_{\mathrm{tot}}\left(q,\left\{\xi_{i}\right\}, t\right)= & H_{q}(q, t)+\sum_{i} \frac{p_{i}^{2}}{2 m_{i}}+\frac{1}{2} \sum_{i j} \Lambda_{i j} \xi_{i} \xi_{j} \\
& -\sum_{i} \xi_{i} f_{i}(q)
\end{aligned}
$$

where $p_{i}$ are momentum coordinates canonical to $\xi_{i}$, and $\Lambda_{i j}$ is the force constant matrix of the bath. This is a more general form of the Caldeira-Leggett Hamiltonian [35] since the environment coupling is a general function of $q$ rather than being strictly bilinear.

In the SLN method the open system and environment density matrices are initialized in a partitioned state where the full density matrix $\rho_{0}=\rho_{\text {tot }}\left(t_{0}\right)$ is the tensor product of the open system density matrix $\boldsymbol{\rho}_{q}\left(t_{0}\right)$ and that of its environment $\boldsymbol{\rho}_{\xi}\left(t_{0}\right)$ at some initial time $t_{0}$,

$$
\rho_{0}=\rho_{q}\left(t_{0}\right) \otimes \rho_{\xi}\left(t_{0}\right)
$$

In principle, the open system and its environment can be initialized in the canonical equilibrium state using the ESLN formalism $[16,18]$, with the system and environment in thermal contact such that they are fully thermalized. However, here we shall limit ourselves to the partitioned initial state (2) and the SLN method.

Tracing over the environment variables $[15,19]$, it is possible to obtain the stochastic Liouville-von Neumann (SLN) equation, an SDE which describes the evolution of a stochastic reduced density matrix for the system driven by complex colored noises, where the physical reduced density matrix is obtained by taking the average over a sample of many 
realizations of the dynamics. This SLN takes the form

$$
\begin{aligned}
i \hbar \frac{d \rho(t)}{d t}= & {\left[H_{q}(t), \rho(t)\right]-\eta(t)[f(q), \rho(t)] } \\
& -\frac{\hbar}{2} v(t)\{f(q), \rho(t)\},
\end{aligned}
$$

where $\rho(t)$ represents the stochastic reduced density matrix and the square (curly) brackets represent standard (anti-) commutators, with the physical reduced density matrix given by $\rho^{p h}(t)=\langle\rho(t)\rangle$. Here $\eta(t)$ and $v(t)$ are the aforementioned complex colored noises, angle brackets $\langle\cdot\rangle$ represent an average over the noises, $H_{q}(t)$ is the open system Hamiltonian mentioned previously (which may depend explicitly on time), and $f(q)$ is the (universal) function which couples the system to the environmental oscillators, assumed to be time independent.

The noises all have zero mean and are otherwise defined by their correlation functions

$$
\begin{aligned}
\left\langle\eta(t) \eta\left(t^{\prime}\right)\right\rangle=\hbar & \int_{0}^{\infty} \frac{d \omega}{\pi} J(\omega) \operatorname{coth}\left(\frac{1}{2} \beta \hbar \omega\right) \cos \left(\omega\left(t-t^{\prime}\right)\right) \\
\equiv & K_{\eta \eta}\left(t-t^{\prime}\right) \\
\left\langle\eta(t) v\left(t^{\prime}\right)\right\rangle= & -2 i \Theta\left(t-t^{\prime}\right) \int_{0}^{\infty} \frac{d \omega}{\pi} J(\omega) \sin \left[\omega\left(t-t^{\prime}\right)\right] \\
\equiv & K_{\eta \nu}\left(t-t^{\prime}\right)=i R\left(t-t^{\prime}\right) \\
& \left\langle v(t) v\left(t^{\prime}\right)\right\rangle=0, \quad \forall t, t^{\prime}
\end{aligned}
$$

where $J(\omega)$ is the spectral density of the environment and $\beta=$ $1 / k_{B} T$ where $T$ is the temperature of the environment. From now on we set $\hbar=1$. In this study we take $J(\omega)$ to be of the Drude form

$$
J(\omega)=\omega\left[1+\left(\frac{\omega}{\omega_{c}}\right)^{2}\right]^{-2},
$$

where the cutoff frequency $\omega_{c}$ controls the decaying character of $J(\omega)$ at large $\omega$, and there is a hard cutoff such that $J\left(\omega>\omega_{c}\right)=0$. To be explicit, the Drude form of the spectral density is used prior to a hard cutoff $\omega_{c}$ that specifies the maximum phonon frequency of the bath above which there is no contribution associated with higher frequencies.

\section{B. Spin-boson model}

Thus far, the system Hamiltonian $H_{q}$ has been kept fully general, as has the form of the system-environment coupling, $f(q)$. We will adopt the spin-boson Hamiltonian for our system of interest, which, in a basis of a generic two-state system, is

$$
\begin{aligned}
H_{q}(t)= & \frac{1}{2} \Delta(t) \sigma_{x}+\frac{1}{2} \epsilon(t) \sigma_{z}=\frac{1}{2} \Delta(t)(|0\rangle\langle 1|+| 1\rangle\langle 0|) \\
& +\frac{1}{2} \epsilon(t)(|0\rangle\langle 0|-| 1\rangle\langle 1|) .
\end{aligned}
$$

Here $\sigma_{x, y, z}$ are the standard Pauli spin matrices with $\sigma_{x}$ flipping the spin from one state to the other with tunneling strength $\Delta(t)$ and $\sigma_{z}$ biasing the energy of states with magnitude $\epsilon(t)$. The system-bath coupling [previously $f(q)$ in Eq. (3)] is $\alpha \sigma_{z}$, where $\alpha$ is the coupling strength between the open system and the environmental oscillators. Equation (3) then becomes

$$
\begin{aligned}
i \frac{d \rho(t)}{d t}= & {[H(t), \rho(t)]-\alpha \eta(t)\left[\sigma_{z}, \rho(t)\right] } \\
& -\frac{1}{2} \alpha v(t)\left\{\sigma_{z}, \rho(t)\right\} .
\end{aligned}
$$

Finally, for the spin-boson Hamiltonian it is straightforward to derive coupled SDEs for the $x$-, $y$-, and $z$-spins and $\operatorname{Tr} \rho(t)$ directly,

$$
\begin{gathered}
\frac{d \sigma_{x}(t)}{d t}=-[\epsilon(t)-2 \alpha \eta(t)] \sigma_{y}(t), \\
\frac{d \sigma_{y}(t)}{d t}=-\Delta(t) \sigma_{z}(t)+[\epsilon(t)-2 \alpha \eta(t)] \sigma_{x}(t), \\
\frac{d \sigma_{z}(t)}{d t}=\Delta(t) \sigma_{y}(t)+i \alpha \nu(t) \operatorname{Tr} \rho(t), \\
\frac{d \operatorname{Tr} \rho(t)}{d t}=i \alpha \nu(t) \sigma_{z}(t) .
\end{gathered}
$$

To be clear, these are expectation values of spins $\sigma_{x, y, z}(t)=$ $\operatorname{Tr}\left(\sigma_{x, y, z} \boldsymbol{\rho}(t)\right)$ obtained from a single realization of the stochastic reduced density matrix. The physical expectation values would then be obtained by the average over many such realizations, $\left\langle\sigma_{x, y, z}(t)\right\rangle$.

\section{NOISE GENERATION SCHEMES}

The correlation functions given by Eqs. (4)-(6) act as constraints on the noise generated but do not uniquely define them, leaving some freedom to specify the generation procedure.

For the purpose of considering different representations of the noises, we adopt the most general form of the linear filtering ansatz [36],

$$
\begin{aligned}
& \eta(t)=\int_{-\infty}^{\infty} d t^{\prime} \sum_{j} F_{j} f_{j}\left(t-t^{\prime}\right) x_{j}\left(t^{\prime}\right), \\
& v(t)=\int_{-\infty}^{\infty} d t^{\prime} \sum_{j} G_{j} g_{j}\left(t-t^{\prime}\right) x_{j}\left(t^{\prime}\right),
\end{aligned}
$$

where the $\left\{f_{j}\right\}$ and $\left\{g_{j}\right\}$ are real functions of time (henceforth referred to as filters) which must be chosen such that the correlation functions of Eqs. (4)-(6) are satisfied. $F_{j}$ and $G_{j}$ are either 1 or the imaginary unit $i$ and are also chosen to be consistent with the correlation functions, and the $\left\{x_{j}\right\}$ are real-valued white Gaussian uncorrelated noises.

\section{A. Orthogonal decomposition}

The form used above has the benefit that it is possible, if desired, to decompose each noise into orthogonal components that are correlated with only one other component $[17,18]$. This orthogonality can, e.g., be achieved by expressing the noises as

$$
\begin{aligned}
\eta(t)= & \int_{-\infty}^{\infty} d t^{\prime} f_{1}\left(t-t^{\prime}\right) x_{1}\left(t^{\prime}\right) \\
& +\int_{-\infty}^{\infty} d t^{\prime} f_{2}\left(t-t^{\prime}\right)\left[x_{2}\left(t^{\prime}\right)+i x_{3}\left(t^{\prime}\right)\right]
\end{aligned}
$$




$$
\begin{aligned}
v(t)= & \int_{-\infty}^{\infty} d t^{\prime} g_{1}\left(t-t^{\prime}\right)\left[i x_{1}\left(t^{\prime}\right)+x_{4}\left(t^{\prime}\right)\right] \\
& +\int_{-\infty}^{\infty} d t^{\prime} g_{2}\left(t-t^{\prime}\right)\left[x_{3}\left(t^{\prime}\right)+i x_{2}\left(t^{\prime}\right)\right],
\end{aligned}
$$

While it is possible to add an arbitrary number of terms of the appropriate form containing pairs of noises as is done here, we consider no more than one such term in the expansion of $\eta(t)$ and up to two in $v(t)$, since this restricts the number of necessary white noises to the minimum possible number. We emphasize that while this does represent a loss of generality compared to Eqs. (14) and (15), there are three benefits. First, autocorrelative and cross-correlative components of the noise can be immediately identified by their structure, with, e.g., the first term of Eq. (16) being autocorrelative while the second term is cross-correlative. Second, the noise can be decomposed into orthogonal components which are co-correlated with only one other component. For example, the term involving $f_{2}$ is correlated only with the term involving $g_{2}$ and no other terms. And, third, forming complex noise from pairs of real noises ensures that their autocorrelation vanishes by construction. This is especially useful for the $v$ noise which has zero self-correlation.

The choice of filters $f_{1}, f_{2}, g_{1}$, and $g_{2}$ is then made by relating the expectation values of the noises to the appropriate correlation functions, Eqs. (4)-(6), and taking Fourier transforms (indicated by a tilde). In particular,

$$
\tilde{K}_{\eta \eta}(\omega)=\tilde{f}_{1}(\omega) \tilde{f}_{1}(-\omega) .
$$

Note that $\tilde{f}^{*}(\omega)=\tilde{f}(-\omega)$ for any real function $f(t)$. Since $K_{\eta \eta}(t)$ is real and even, its Fourier transform is also real and even, so $\tilde{K}_{\eta \eta}(\omega)=\tilde{K}_{\eta \eta}(-\omega)$, and thus it is convenient to choose $\tilde{f}_{1}(\omega)$ to be real, hence

$$
\tilde{K}_{\eta \eta}(\omega)=\tilde{f}_{1}(\omega)^{2} \Rightarrow \tilde{f}_{1}(\omega)=\sqrt{\tilde{K}_{\eta \eta}(\omega)},
$$

thus specifying the autocorrelative filter, $\tilde{f}_{1}(\omega)$.

The correlation between $\eta$ and $v, K_{\eta \nu}(t)$ of Eq. (20), requires that the following constraint in Fourier space be satisfied:

$$
\tilde{f}_{1}(\omega) \tilde{g}_{1}(-\omega)+2 \tilde{f}_{2}(\omega) \tilde{g}_{2}(-\omega)=\tilde{R}(\omega),
$$

where $R(t)=-i K_{\eta v}(t)$ [Eq. (5)]; note that $R(t)$ is a real function. Derivations of the Fourier transforms $\tilde{K}_{\eta \eta}(\omega)$ and $\tilde{K}_{\eta v}(\omega)$ and their properties are provided in Appendixes A 1 and A 2. The three filters $\tilde{g}_{1}(\omega), \tilde{f}_{1}(\omega)$, and $\tilde{f}_{2}(\omega)$ are determined by only a single condition [Eq. (20)], and hence their full specification is subject to different possible choices, some of which we now discuss.

\section{Delta scheme}

Choosing $g_{1}$ to be zero and $g_{2}(t)$ to be a $\delta$ function, gives

$$
\begin{gathered}
f_{2}(t)=-\frac{i}{2} K_{\eta v}(t), \\
g_{2}(t)=\delta(t) .
\end{gathered}
$$

This choice can be reversed by switching the $\delta$ function around. For obvious reasons, we refer to this as the delta choice; it was made in previous work [17].

\section{Constrained choice}

Taking the constraint Eq. (20) and setting $\tilde{f}_{2}$ and $\tilde{g}_{2}$ to be zero, this becomes a decomposition with $\tilde{f}_{1}$ given by Eq. (19) and $\tilde{g}_{1}$ given by

$$
\tilde{g}_{1}(\omega)=\frac{\tilde{R}(-\omega)}{\sqrt{\tilde{K}_{\eta \eta}(\omega)}} .
$$

We refer to this as the constrained choice, since the two filters $\tilde{f}_{1}(\omega)$ and $\tilde{g}_{1}(\omega)$ are fully constrained (defined) with no flexibility.

\section{Like scheme}

In a similar fashion, $\tilde{g}_{1}(\omega)$ can be set to zero instead of $\tilde{f}_{2}(\omega)$ and $\tilde{g}_{2}(\omega)$, in which case Eq. (20) becomes

$$
\tilde{f}_{2}(\omega) \tilde{g}_{2}(-\omega)=\frac{1}{2} \tilde{R}(\omega) .
$$

A possible choice for $\tilde{f}_{2}(\omega)$ and $\tilde{g}_{2}(\omega)$ is to require that $\tilde{f}_{2}(\omega)=\tilde{g}_{2}(-\omega)$ such that

$$
\tilde{f}_{2}(\omega)=\sqrt{\frac{1}{2} \tilde{R}(\omega)}=\sqrt{-\frac{i}{2} \tilde{K}_{\eta \nu}(\omega),}
$$

with $\tilde{g}_{2}(\omega)$ simply given by sending $\omega \rightarrow-\omega$ on the right hand side. For obvious reasons, we refer to this choice as the like choice; it has been used by us previously [18].

\section{Reduced scheme}

Any combination of the like and constrained choices will also be allowed, since they would satisfy the general definitions of the noises (16) and (17). We introduce a set of filters $\tilde{f}_{1}, \tilde{f}_{2}, \tilde{g}_{1}$, and $\tilde{g}_{2}$ which utilize both of the above choices via the introduction of an auxiliary mixing function $\tilde{A}(\omega)$,

$$
\begin{gathered}
\tilde{f}_{1}(\omega)=\sqrt{\tilde{K}_{\eta \eta}(\omega),} \\
\tilde{f}_{2}(\omega)=\sqrt{\frac{1}{2} \tilde{A}(\omega) \tilde{R}(\omega),} \\
\tilde{g}_{1}(\omega)=\frac{\tilde{R}(-\omega)}{\sqrt{\tilde{K}_{\eta \eta}(\omega)}}[1-\tilde{A}(-\omega)], \\
\tilde{g}_{2}(\omega)=\sqrt{\frac{1}{2} \tilde{A}(-\omega) \tilde{R}(-\omega) .}
\end{gathered}
$$

Here the mixing function $\tilde{A}(\omega)$ controls which of the two choices (like and/or constrained) is being used at each value of $\omega$, and it is easy to verify that these filters satisfy Eq. (20). A similar expression was recently presented [20], though due to incorrect definitions of the filters it was neither general nor correct, as the properties of the Fourier transforms (see Appendix A) were not satisfied in any case except for the autocorrelative component of $\eta$, which is already fully determined. The special cases of $\tilde{A}(\omega)=0$ and $\tilde{A}(\omega)=1$ correspond to the constrained and like choices, respectively.

By examination of the evolution of $\operatorname{Tr}(\rho(t))$ [Eq. (13)], it is clear that the non-Hermitian (trace nonpreserving) dynamics of the stochastic density matrix is driven solely by $v$. The spread of values of the trace will grow with time, just as the variance of the displacement of a Brownian walker grows with time, and this spreading requires an ever larger ensemble of 
realizations for the average trace to remain close to unity at late times. We thus try to choose the mixing function $\tilde{A}(\omega)=$ $\{0,1\}$ to reduce the average amplitude of $v(t)$, noting that

$$
\begin{aligned}
\left\langle|v(t)|^{2}\right\rangle= & \int \frac{d \omega}{2 \pi}\left\{2 \frac{|\tilde{R}(-\omega)|^{2}}{\tilde{K}_{\eta \eta}(\omega)}|1-\tilde{A}(-\omega)|^{2}\right. \\
& +|\tilde{R}(-\omega)||\tilde{A}(-\omega)|\} .
\end{aligned}
$$

We choose $\tilde{A}(\omega)=0$ when the first term in the integrand is smaller than the second term; otherwise $\tilde{A}(\omega)$ should be 1 , that is (cf. Ref. [20]),

$$
\tilde{A}(\omega)= \begin{cases}0, & \text { when }|\tilde{R}(-\omega)|^{2} / \tilde{K}_{\eta \eta}(\omega) \leqslant|\tilde{R}(-\omega)| \\ 1, & \text { otherwise. }\end{cases}
$$

This choice, which can be done individually for every value of $\omega$, should then significantly reduce the average magnitude of $v(t)$, diminishing the impact of the non-Hermitian dynamics and improving the convergence of the ensemble average. Thus we refer to this as the reduced choice.

\section{Optimized scheme}

This naturally leads us to choosing the optimal mixing function $\tilde{A}(\omega)$ which truly minimizes the average magnitude of $v$; this is the $v$-optimized choice. Starting with Eq. (20), it can be shown that the mixing function must be real and even (see Appendix B). By setting the derivative of Eq. (30) with respect to $\tilde{A}(\omega)$ equal to zero, we find the $v$-optimized mixing function to be

$$
\tilde{A}(\omega)=1-\frac{\tilde{K}_{\eta \eta}(\omega)}{4|\tilde{R}(\omega)|} .
$$

Substituting this $\tilde{A}(\omega)$ into Eqs. (27) and (29) gives the corresponding filters as

$$
\begin{gathered}
\tilde{f}_{2}(\omega)=\sqrt{\frac{\tilde{R}(\omega)}{2}\left[1-\zeta \frac{\tilde{K}_{\eta \eta}(\omega)}{|\tilde{R}(\omega)|}\right]} \\
\tilde{g}_{1}(\omega)=\zeta \frac{\tilde{R}(-\omega)}{|\tilde{R}(\omega)|} \sqrt{\tilde{K}_{\eta \eta}(\omega)}, \\
\tilde{g}_{2}(\omega)=\sqrt{\frac{\tilde{R}(-\omega)}{2}\left[1-\zeta \frac{\tilde{K}_{\eta \eta}(\omega)}{|\tilde{R}(\omega)|}\right]},
\end{gathered}
$$

with $\tilde{f}_{1}(\omega)=\sqrt{\tilde{K}_{\eta \eta}(\omega)}$ as before, and $\zeta=1 / 4$.

An alternative approach would be to minimize $\left\langle|\eta(t)|^{2}\right\rangle+$ $\left\langle|v(t)|^{2}\right\rangle$ rather than just the average magnitude of $v$, by considering

$$
\begin{aligned}
& \left\langle|\eta(t)|^{2}\right\rangle+\left\langle|v(t)|^{2}\right\rangle \\
& =\int \frac{d \omega}{2 \pi}\left\{\tilde{K}_{\eta \eta}(\omega)+|\tilde{R}(\omega)|[|\tilde{A}(\omega)|\right. \\
& \left.\quad+|\tilde{A}(-\omega)|]+2 \frac{|\tilde{R}(\omega)|^{2}}{\tilde{K}_{\eta \eta}(\omega)}|1-\tilde{A}(-\omega)|^{2}\right\}
\end{aligned}
$$

for which the minimizing mixing function is

$$
\tilde{A}(\omega)=1-\frac{\tilde{K}_{\eta \eta}(\omega)}{2|\tilde{R}(\omega)|},
$$

with its own $\tilde{f}_{2}, \tilde{g}_{1}$, and $\tilde{g}_{2}$, which are defined by the same Eqs. (33)-(35), but with $\zeta=1 / 2$. We refer to this as the $\eta v$ optimized scheme.

The derivations of the minimizing mixing function for both optimized choices are presented in Appendix B.

It is important to stress that minimizing the combined magnitude $\left\langle|\eta(t)|^{2}\right\rangle+\left\langle|v(t)|^{2}\right\rangle$ will not necessarily minimize the variance of the trace, nor the rate of its exponential growth. As far as we are aware it is not possible to analytically minimize the growth of the trace directly, so we are forced to approach any optimization via an ansatz, in this case by introducing the mixing function and making use of the freedom in its definition. While the optimal mixing functions derived here affect the properties of the noises as intended, they do not guarantee that the behavior of the trace will be modified in the desired way for all parameters or over all timescales. This approach should be thought of as an indirect optimization of the properties of the dynamics.

\section{Dynamical rescaling}

It is possible to go one step further by introducing a dynamical rescaling of the cross-correlative filters $\tilde{f}_{2}$ and $\tilde{g}_{2}$, as was done for the like scheme in previous work [18]. This type of scaling was first introduced for autocorrelative colored noises in Ref. [37], and expanded to cross-correlative noises in Ref. [18]. Since dividing $\tilde{f}_{2}(\omega)$ by an arbitrary $\omega$-dependent factor $\tilde{\chi}(\omega)$ and multiplying $\tilde{g}_{2}(\omega)$ by the same factor will leave the correlation $K_{\eta \nu}$ between $\eta$ and $v$ unchanged, we can choose this factor optimally. However, attempting to minimize $\left\langle|\eta(t)|^{2}\right\rangle+\left\langle|v(t)|^{2}\right\rangle$ with respect to $\tilde{\chi}(\omega)$ in Fourier space for each $\omega$ gives the result that $\tilde{\chi}(\omega)= \pm 1, \pm i$, which is trivial.

As stated above, while this is the $\tilde{\chi}(\omega)$ which minimizes the combined magnitude of the noises, it is more desirable to minimize the growth of the trace directly. For this reason we consider a similar scaling in the time domain, instead dividing $f_{2}(t)$ by a scaling factor and multiplying $g_{2}(t)$ by that same number, even though the scaling freedom is most apparent in Fourier space. We can then choose the scaling factor to minimize the rate of spreading of $|\operatorname{Tr} \rho(t)|$. We do this by sampling the final value of the trace for a range of scaling factors and minimizing the standard error in the mean trace. Note that where the optimization of the mixing function $\tilde{A}$ was analytical, choosing this optimal scaling is a numerical procedure.

It is convenient to implement this scaling via the ratio between the noises generated using $f_{2}$ and $g_{2}$ before any scaling is applied, denoted here as $\eta_{0}$ and $v_{0}$, respectively. The scaled noises are then obtained from the unscaled noises as $\eta^{\text {new }}=\lambda_{\nu \eta} \eta_{0}$ and $v^{\text {new }}=v_{0} / \lambda_{\nu \eta}$, where

$$
\lambda_{\nu \eta}=\sqrt{\lambda} \sqrt{\frac{\sum_{n}\left|v_{0}\left(t_{n}\right)\right|}{\sum_{n}\left|\eta_{0}\left(t_{n}\right)\right|}}
$$

and $\lambda$ is a parameter (to be determined) representing the desired ratio between $v^{\text {new }}$ and $\eta^{\text {new }}$. Here the sums are over a single realization of the noises in time, adding the value of the noise at each discrete time, $t_{n}$. 


\section{B. Convex optimized scheme}

It is also possible to optimize the noise generation scheme in a different manner using the general form of the noises (14) and (15), without explicitly introducing a mixing function [21]. Instead of minimizing the average of the square magnitude of $v$ or the sum of square magnitudes of $v$ and $\eta$, the sum of the imaginary parts of $\eta$ and $v$ can be minimized, subject to the correlations, by the method of convex optimization. We can reproduce the analytical expression obtained in Ref. [21] for the correlations of the real and imaginary components of the noises $\eta$ and $v$ using the following forms:

$$
\begin{gathered}
\eta(t)=\int_{-\infty}^{\infty} d t^{\prime} f_{1}\left(t-t^{\prime}\right) x_{1}\left(t^{\prime}\right)+i \int_{-\infty}^{\infty} d t^{\prime} f_{2}\left(t-t^{\prime}\right) x_{2}\left(t^{\prime}\right) \\
v(t)=\int_{-\infty}^{\infty} d t^{\prime} g_{1}\left(t-t^{\prime}\right)\left[x_{1}\left(t^{\prime}\right)+i x_{2}\left(t^{\prime}\right)\right]
\end{gathered}
$$

The filters in Fourier space can be written as

$$
\begin{aligned}
& \tilde{f}_{1}(\omega)=\frac{1-\tilde{C}(\omega)}{\sqrt{1-2 \tilde{C}(\omega)}} \sqrt{\tilde{K}_{\eta \eta}(\omega)}, \\
& \tilde{f}_{2}(\omega)=\frac{\tilde{C}(\omega)}{\sqrt{1-2 \tilde{C}(\omega)}} \sqrt{\tilde{K}_{\eta \eta}(\omega)}, \\
& \tilde{g}_{1}(\omega)=\sqrt{1-2 \tilde{C}(\omega)} \frac{\tilde{R}(-\omega)}{\sqrt{\tilde{K}_{\eta \eta}(\omega)}},
\end{aligned}
$$

where

$$
\tilde{C}(\omega)=\frac{1}{2}\left\{1-\left[\frac{4|\tilde{R}(\omega)|^{2}}{\tilde{K}_{\eta \eta}(\omega)^{2}}+1\right]^{-1 / 2}\right\} .
$$

\section{Deconvolution for reduced and constrained schemes}

Division in Fourier space can introduce troublesome amplification for frequencies near which the denominator is close to zero [34,38] [see, for example, Eq. (23)]. The $\tilde{C}(\omega)$ function in the convex optimized scheme removes explicit divisions where this would occur and can be implemented as it stands, as it involves only division by $4|\tilde{R}(\omega)|^{2}+\tilde{K}_{\eta \eta}(\omega)^{2}$. The same applies to the $\nu$-optimized and $\eta \nu$-optimized schemes where the filters remain finite since $\tilde{R} /|\tilde{R}|$ has real and imaginary parts which are bounded by \pm 1 . Thus the constrained and reduced schemes are the only schemes which include explicit division by a filter in Fourier space, in this case by $\sqrt{\tilde{K}_{\eta \eta}(\omega)}$ in Eqs. (23) and (28), so they require additional care.

This issue of frequency amplification around the zeros of $\sqrt{\tilde{K}_{\eta \eta}(\omega)}$ can be eased by deconvolution methods. A deconvolution is the inverse operation to a convolution which can be naively interpreted as division in Fourier space. In practice, the process is more complex. Even for two deterministic functions, there is always an issue of division close to zero, or of rounding errors which can cause numerical instabilities in the deconvolved signal after taking the inverse Fourier transform $[33,34]$. In particular, these instabilities can depend on properties of the signal such as its length $t_{\max }$ and spacing
$\Delta t$, since these affect the sensitivity of the Fourier transform to small numbers.

We adopt the deconvolution method of Wiener filtering [39] which minimizes the mean square error between some desired quantity $q(t)$ to be determined and its estimate $\hat{q}(t)$. Considering the signal associated with $q(t)$ to be

$$
y(t)=\int d t^{\prime} h\left(t-t^{\prime}\right) q\left(t^{\prime}\right)+\xi(t),
$$

where $h(t)$ is the known response function of $q(t)$ and $\xi(t)$ is some unknown noise, the estimate of the signal in the time domain is

$$
\hat{q}(t)=\int d t^{\prime} w\left(t-t^{\prime}\right) y\left(t^{\prime}\right)
$$

where we have introduced some "inverse" to the response function, $w(t)$. In Fourier space this becomes

$$
\tilde{q}(\omega)=\tilde{W}(\omega) \tilde{Y}(\omega),
$$

with

$$
\tilde{W}(\omega)=\frac{\tilde{H}^{*}(\omega)}{|\tilde{H}(\omega)|^{2}+\frac{1}{\mathrm{SNR}}}
$$

being the Fourier transform of the inverse response function $w(t)$. Here $\tilde{H}(\omega)$ and $\tilde{Y}(\omega)$ are the Fourier transforms of $h(t)$ and $y(t)$, respectively. This $\tilde{W}(\omega)$ is known as the Wiener filter and is used as an estimate of $\tilde{H}(\omega)$ with the problematic frequency amplification removed. It arises directly from minimizing the mean square error $\mathbb{E}|\hat{q}(t)-q(t)|^{2}[38,40]$. Finally, SNR is the signal to noise ratio, or, more concretely, it is the ratio between the mean power spectral densities of the signal and the noise. Typically for the Wiener filter, the SNR needs to be estimated in some way, especially when the form of the noise $\xi(t)$ is not exactly known $[38,40]$ and is usually chosen to be a constant value such that the signal is guaranteed to be larger than the noise.

Adopting this method, the division by $\sqrt{\tilde{K}_{\eta \eta}}$ in the constrained and reduced schemes should be replaced with multiplication by the corresponding Wiener filter,

$$
\frac{1}{\sqrt{\tilde{K}_{\eta \eta}(\omega)}} \rightarrow \frac{\sqrt{\tilde{K}_{\eta \eta}(\omega)}}{\tilde{K}_{\eta \eta}(\omega)+\gamma \max _{\omega}\left|\sqrt{\tilde{K}_{\eta \eta}(\omega)}\right|}
$$

with a signal-to-noise ratio $\mathrm{SNR}=\left[\gamma \max _{\omega}\left|\sqrt{\tilde{K}_{\eta \eta}(\omega)}\right|\right]$ where $\gamma$ is a small parameter. This allows the correction term to vary depending on the simulation time $t_{\max }$, and to stabilize the division while still remaining small. Note that this is something of a numerical fix; it will modify the correlation function $\left\langle\eta(t) v\left(t^{\prime}\right)\right\rangle$ so that it no longer matches the desired correlation $K_{\eta \nu}\left(t-t^{\prime}\right)$ [Eq. (5)] exactly, though the introduction of the small parameter $\gamma$ allows us to control the size of this deviation.

\section{Deconvolution and causality}

The instability of the direct Fourier division method can be observed by investigating the behavior of the noises for different lengths of the simulation, $t_{\max }$. We compare in Fig. 1 

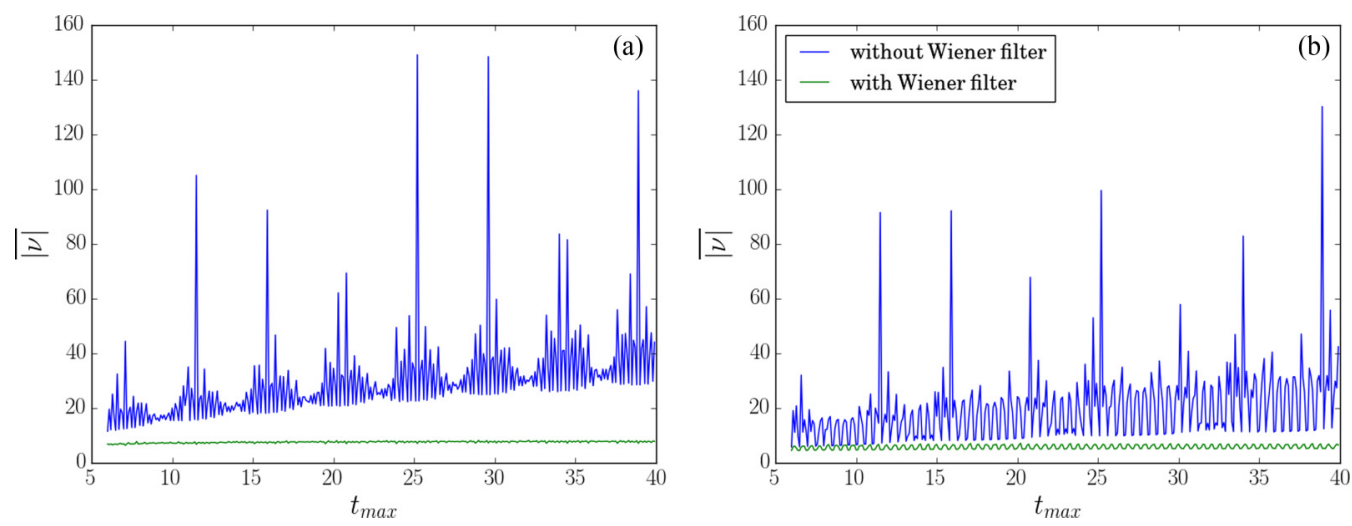

FIG. 1. Average magnitude of $v(t)$ taken across 500 realizations for each $t_{\max }$ for (a) the constrained and (b) reduced schemes with (green) and without (blue) the Wiener filter using $\gamma=0.01, \beta=1, \Delta=1, \epsilon=-1, \alpha=0.05$, and $\omega_{c}=25$.

the stability of these two schemes with and without the Wiener filter, by observing the average magnitude of $v(t)$ for different values of $t_{\max }$. The application of the Wiener filter to the reduced and constrained noise schemes improves their stability enormously, in some cases by as much as an order of magnitude, and significantly weakens the erroneous dependence of $v$ on $t_{\max }$, though not removing it entirely. The trade-off for this improvement is a violation of the $\eta-v$ correlation function by introducing a breakdown of causality, as can be seen in Fig. 2.

The application of deconvolution methods thus successfully stabilizes the $t_{\max }$ dependence of $v$, decreasing its average magnitude by reducing the power of frequencies around the singularities in its spectral density. This improves the convergence and maximum possible run time of the dynamics, at the cost of weakening causality in the $\eta-v$ correlation. Weakening the Heaviside function or removing it entirely by hand also has this effect of smoothing the $v$ noise

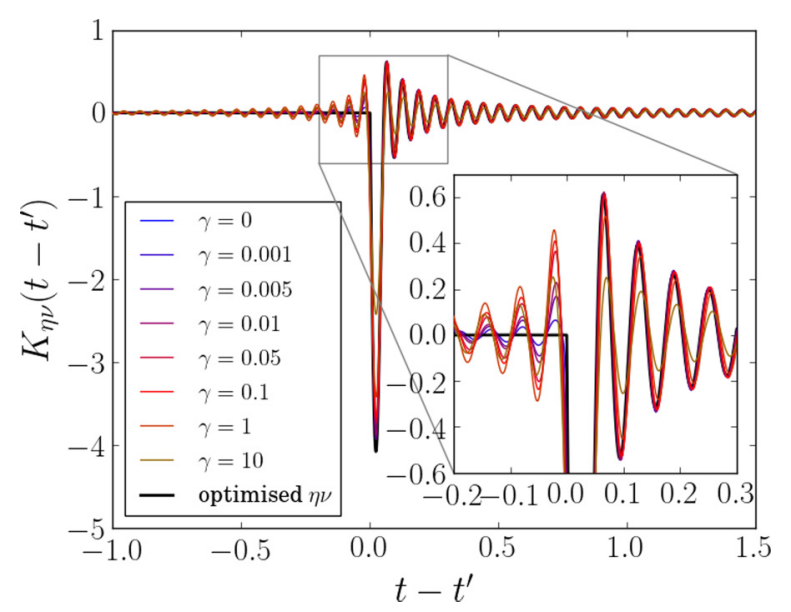

FIG. 2. The $\eta-v$ correlation function with different values of the parameter $\gamma$ in the Wiener filter for the constrained noise scheme. The $\eta v$ optimized scheme overlaps with the desired correlation $K_{\eta v}$ such that $K_{\eta v}$ could not be seen, so it is not shown. $\beta=1, t_{\max }=12$, $d t=0.01, \omega_{c}=25, \alpha=0.05$ for $10^{4}$ realizations. The zoomed inset highlights the region in which the symmetrization of the correlation as $\gamma$ increases can be clearly seen. and reducing the likelihood of realizations which contain atypically large values, in turn improving convergence.

While the causality of $K_{\eta v}$ is a requirement of the theory, the introduction of the $\gamma$ parameter gives us a method of deconvolution for which we can ensure any deviation from the theory is well controlled.

We have carried out tests of the above implementation of deconvolution. In Fig. 3 we show the dynamics of the $z$-spin $\left\langle\sigma_{z}(t)\right\rangle$ with a constant Hamiltonian [the relaxation to the equilibrium case, Fig. 3(a)] and a Landau-Zener sweep [nonequilibrium case, 3(b)]. The Landau-Zener sweep consists of a linear driving of the form $\epsilon(t)=\kappa t$, and has a known analytic solution in the $t \rightarrow \infty$ limit when the system was initialized in the ground state $|1\rangle$ in the infinite past at zero temperature [41]. This limit is [10,41-45]

$$
\left\langle\sigma_{z}\right\rangle_{\mathrm{LZ}}=2 \exp \left\{-\frac{\pi \Delta^{2}}{2 \hbar \kappa}\right\}-1,
$$

and though originally derived for an isolated spin, it has since been shown that the same asymptotic behavior is valid for a dissipative spin coupled to a harmonic environment at zero temperature, when the coupling is provided entirely via $\sigma_{z}$ [46]. Note that this assumes that the system was initialized in the infinite past, whereas here it was initialized at $t=-5$. This is taken into account by modifying the limit appropriately [18], though there is still some deviation associated with the fact that the bath is not at zero temperature and that the limit is asymptotic while the simulation time remains finite.

We expect to recover the canonical equilibrium state (associated with the constant Hamiltonian) [18] and the Landau-Zener limit as known solutions at long times in the two cases, and we investigate the constrained scheme with the Wiener filter for a range of $\gamma$ values, using the $\eta \nu$-optimized scheme which minimizes the sum of magnitudes of $\eta$ and $v$ as a reference. Without the Wiener filter $(\gamma=0)$, the constrained scheme diverges almost immediately for both test cases, whereas for very small $\gamma=0.001$ there is already an improvement, with the accessible simulation time increasing by $\sim 5$ times before $\left\langle\sigma_{z}(t)\right\rangle$ diverges. Note that the behavior of the $z$-spin after divergence is omitted for clarity as it oscillates wildly within an exponentially growing envelope. As $\gamma$ increases to $\sim 0.01$ and then to $\sim 0.1$, the constrained 

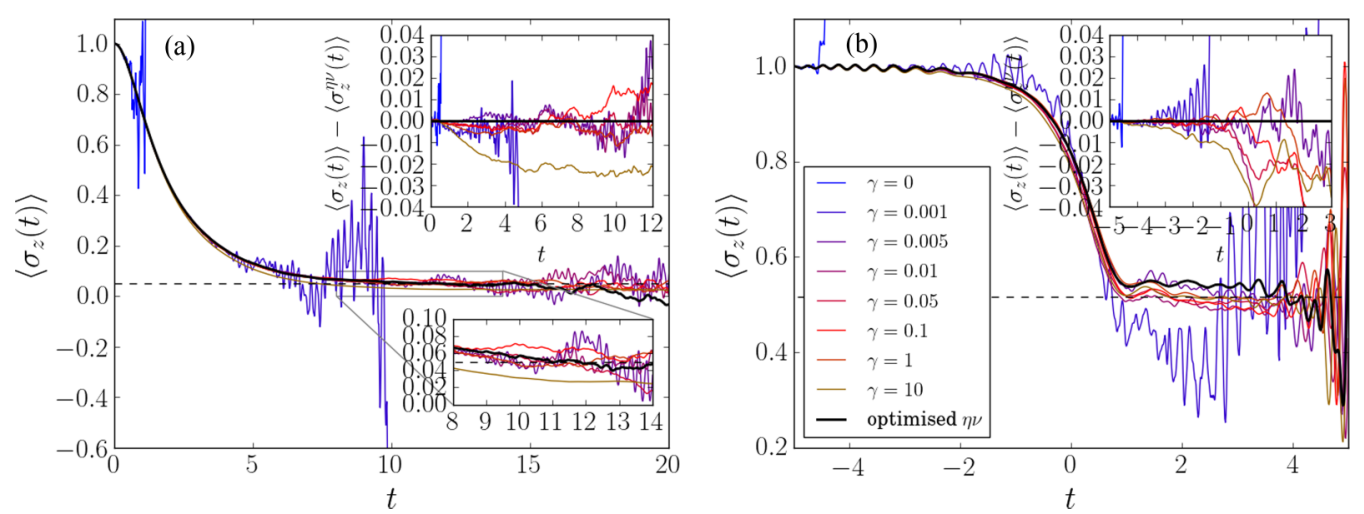

FIG. 3. Comparison of the calculated expectation of the $z$-spin $\left\langle\sigma_{z}(t)\right\rangle$ using the constrained scheme with a range of $\gamma$ values to control deconvolution, and the $\eta v$-optimized scheme $\left\langle\sigma_{z}^{\eta v}(t)\right\rangle$, which minizes the combined magnitude of $\eta$ and $v$. (a) The system is initialized with the $z$-spin being 1 and all other spins being zero and is evolved in time with a constant Hamiltonian, so that the $z$-spin relaxes to its equilibrium value. The canonical equilibrium value of 0.05 for the spin, obtained using the imaginary time evolution methods outlined in Ref. [18], is shown (dashed line) to confirm the validity of the optimized scheme. The inset shows the deviation of the $z$-spin for the constrained scheme from the optimized one. A zoomed-in area at the final stages of equilibration shows the $z$-spin in detail. (b) A Landau-Zener sweep with a time-dependent Hamiltonian where the system is driven linearly with $\epsilon(t)=5 t$, approaching a known asymptotic limit (dashed line). The system is initialized at $t=-5$ with the $z$-spin equal to 1 and all other spins being zero, using the modified Landau-Zener limit of 0.516 to account for the finiteness of the simulation window as outlined in [18]. For clarity, data are no longer plotted once they exceed the vertical scales shown, as the solution becomes unstable and grows exponentially. $\beta=0.1, d t=10^{-2}, \Delta=1, \epsilon=-1, \alpha=0.05$, and $\omega_{c}=25$ for $10^{6}$ realizations.

schemes begin to converge well, more closely resembling the $\eta v$-optimized scheme result $\left\langle\sigma_{z}^{\eta \nu}(t)\right\rangle$ as can be seen in the insets of Fig. 3 where the difference between them is shown. The statistical convergence is best for larger values of $\gamma$, most noticeably for $\gamma=10$, though such a strong Wiener filter introduces a significant deviation from the $\eta v$-optimized scheme and the known solutions, as can clearly be seen in both the inset and zoomed region in Fig. 3(a). The same is true in the nonequilibrium Landau-Zener case, Fig. 3(b), where for smaller $\gamma$ the $z$-spin converges poorly while for larger $\gamma$ it converges better at the expense of introducing a deviation from the solution used as a reference. Thus a compromise value of $\gamma$ must be chosen.

The best $\gamma$ value can be chosen by computing the integrated absolute deviation, $\int d t^{\prime}\left|\left\langle\sigma_{z}(t)\right\rangle-\left\langle\sigma_{z}^{\eta \nu}(t)\right\rangle\right|$, for the data ranges shown in the insets of Fig. 3, presented in Fig. 4. This can be thought of as the total deviation from the $\eta v-$ optimized scheme within the region where the convergence of the schemes are comparable, with results for $\gamma=0$ and 0.001 not shown since they do not remain well converged on useful timescales. The $\gamma$ which minimizes this quantity is the one with the smallest deviation from the correct dynamics which we find to be $\gamma=0.01$ for both the constant Hamiltonian and Landau-Zener cases. By minimizing this deviation, we ensure that the breakdown of causality is well controlled while still managing to correctly handle the deconvolution and improve the convergence of the system properties.

\section{RESULTS}

\section{A. Verifying SLN dynamics with a quantum nondemolition model}

In this section, we verify the validity of the SLN equation by comparing the numerical results for $\rho(t)$ simulated using the $\eta v$-optimized scheme with the analytical result obtained for a quantum nondemolition model [47]. The model considered [6] is a zero-temperature model with $H_{s}=-\frac{1}{2} \sigma_{z}$, the coupling to the environment is given by $f=\sigma_{z}$, and the environment's correlation function is taken to be $K(t)=$ $\frac{1}{2} \exp \{-2|t|+i t\}$. Since $f$ and the Hamiltonian commute, the coupling can be thought of as an ideal projective measurement of the open system so as to not disturb its energy [48]. This model can be described exactly by the deterministic master

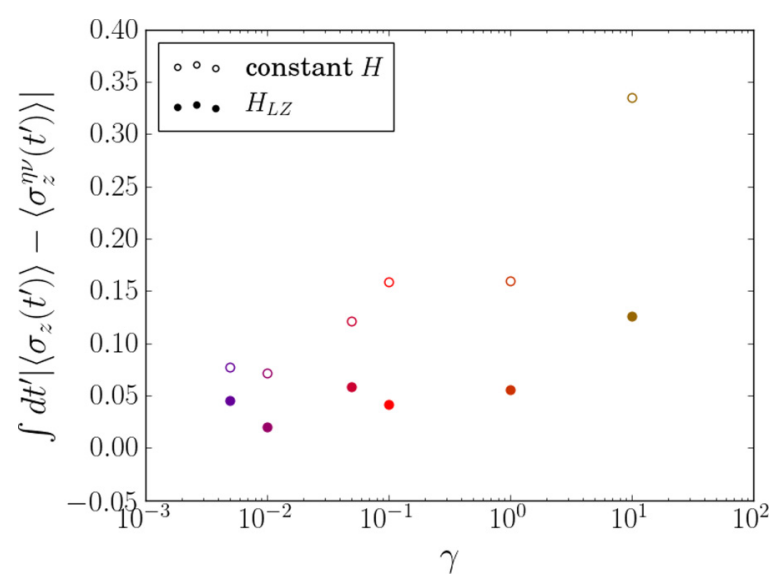

FIG. 4. The total absolute deviation of the dynamics produced using the constrained scheme for different $\gamma$ from the dynamics produced using the $\eta v$-optimized scheme. Values were calculated using the data shown in the insets of Figs. 3(a) and 3(b). Results for $\gamma=0$ and 0.001 are not shown because the dynamics is diverging so the deviation is very large. The value of $\gamma$ which minimizes the total deviation for both the constant Hamiltonian [open circles, Fig. 3(a)] and the Landau-Zener sweep [filled circles, Fig. 3(b)] is 0.01 . 

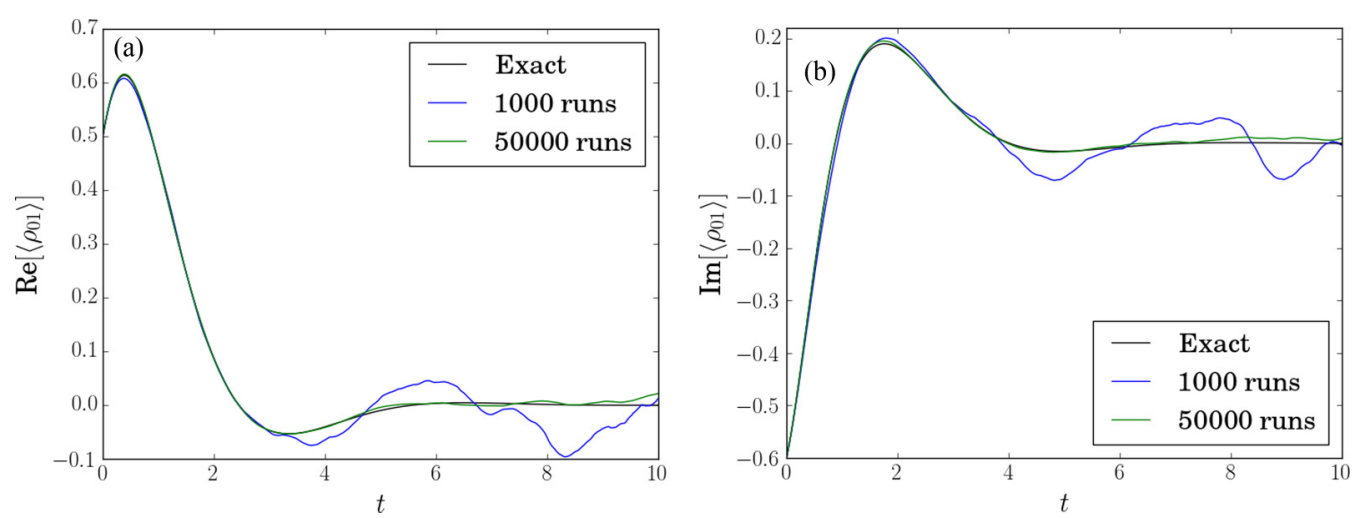

FIG. 5. Dynamics of the (01) element of the reduced density matrix according to the quantum nondemolition model, showing the real part $\operatorname{Re}\left[\left\langle\rho_{01}(t)\right\rangle\right]$ in (a), and the imaginary part $\operatorname{Im}\left[\left\langle\rho_{01}(t)\right\rangle\right]$ in (b). The exact solution (black line) is compared to the SLN numerical solutions for 1000 (blue line) and 50000 (green line) realizations, using the $\eta \nu$-optimized scheme with optimal scaling $\lambda=0.5$ (see Sec. IV C).

equation $[4,49]$

$$
\begin{aligned}
i \frac{d\langle\boldsymbol{\rho}(t)\rangle}{d t}= & {\left[H_{s},\langle\boldsymbol{\rho}(t)\rangle\right]-i C_{r}(t)[f,[f,\langle\boldsymbol{\rho}(t)\rangle]] } \\
& +C_{i}(t)\left[f^{2},\langle\boldsymbol{\rho}(t)\rangle\right],
\end{aligned}
$$

where $C_{r / i}(t)=\int_{0}^{t} d \tau K_{r / i}(t-\tau)$ with $K_{r}(t)=\operatorname{Re}[K(t)]$ and $K_{i}(t)=\operatorname{Im}[K(t)]$. The analytical solution of Eq. (48) is easily found and can be compared to SLN dynamics computed numerically with any of the noise schemes we have considered above, and with correlations $K_{\eta \eta}=\operatorname{Re}[K(t)]$ and $K_{\eta v}=$ $2 i \operatorname{Im}[K(t)]$.

The SLN dynamics using the $\eta \nu$-optimized scheme is shown in Fig. 5, along with the analytical solution of Eq. (48), using the initial condition $\left\langle\boldsymbol{\rho}\left(t_{0}\right)\right\rangle=0.5 I+0.5 \sigma_{x}+0.6 \sigma_{y}$.

It is clear that the numerical simulation for a stochastic average of 50000 realizations matches the analytical solution for the real and imaginary parts of the density matrix element $\left\langle\rho_{01}(t)\right\rangle$ very well. This off-diagonal element is rapidly damped to zero as the environment induces dephasing, with the SLN exhibiting good convergence beyond the initial dephasing and into the equilibrium regime. Additionally, for a much smaller sample of only 1000 realizations, the SLN captures the exact dynamics well for short timescales $t \lesssim 2$. Having verified the validity of the SLN equation, in the next section we investigate the numerical efficiency of the noise schemes introduced in Sec. III.

\section{B. Error control}

The purpose of the optimization schemes developed here is to minimize the typical amplitude of the $v$ noise, since it drives the (potentially) exponential growth of the trace of the stochastic density matrix [Eq. (13)]. This should increase the accessible simulation time (after which convergence is destroyed by numerical blow up), and reduce the variance of observables.

Without some kind of optimization, naive choices such as the delta scheme (Sec. III A 1) in which one of the components of $\eta$ or $v$ is purely white noise, tend to perform badly, or even be entirely pathological. The inclusion of white noise whose variance is one or two orders of magnitude greater than the trace $\operatorname{Tr}(\rho) \sim 1$ requires an excessive number of realizations $\gtrsim 10^{6}$ for the correlation functions (4)-(6) to converge [17], though this by itself does not guarantee well behaved physical dynamics. Instead, the dynamics of the trace (or observables) is highly unstable even on very short timescales, being equally likely to diverge to $+\infty$ as to $-\infty$. The physical average of such diverging observables will thus tend to zero as the white noise dominates the dynamics, effectively drowning out the coupling to the environment via the colored noise. It is also clear that any attempt to normalize with the trace when an instability of this kind has occurred is inappropriate, requiring both division by zero as well as by very large numbers $[15,18]$. For these reasons we do not present any data for the delta scheme, and simply remark that this choice of noise generation scheme is entirely pathological and should not be used, providing an excellent illustration that it is not sufficient merely to satisfy the necessary correlation functions when driving systems using an SLN framework.

The other schemes all mark a drastic improvement on the naive delta scheme, as is seen from Fig. 6. Recall that the like scheme (Sec. III A 3) and constrained scheme (Sec. III A 2) represent the two distinguishing choices, where $\eta$ and $v$ have cross-correlated orthogonal components, or where all correlations are determined by $f_{1}$ and $g_{1}$ only, respectively. The optimized choices, barring convex optimization, rely on weighting these choices to reduce the variance of the trace and extend the duration of stable dynamics.

Relative performance of the schemes is illustrated in Fig. 6, where we show the mean of the magnitude of the trace $|\operatorname{Tr} \rho(t)|$ [Figs. 6(a)-6(c)], its variance Figs. 6(d)-6(f)], and the standard error of the mean [Figs. 6(g)-6(i)] for all the schemes at three inverse temperatures, $\beta=0.1,1,10$. In particular, the performance of the SLN can be quantified via the extent to which the behavior of the average trace of the reduced density matrix remains constant and close to unity, indicating that the dynamics are physical and well converged, shown in Figs. 6(a)-6(c). The physical situation is the same as in Fig. 3(a), where the system is initialized in the state $|1\rangle$ with the $z$-spin equal to 1 and all other spins being zero and relaxes towards the equilibrium state associated with a constant Hamiltonian. 

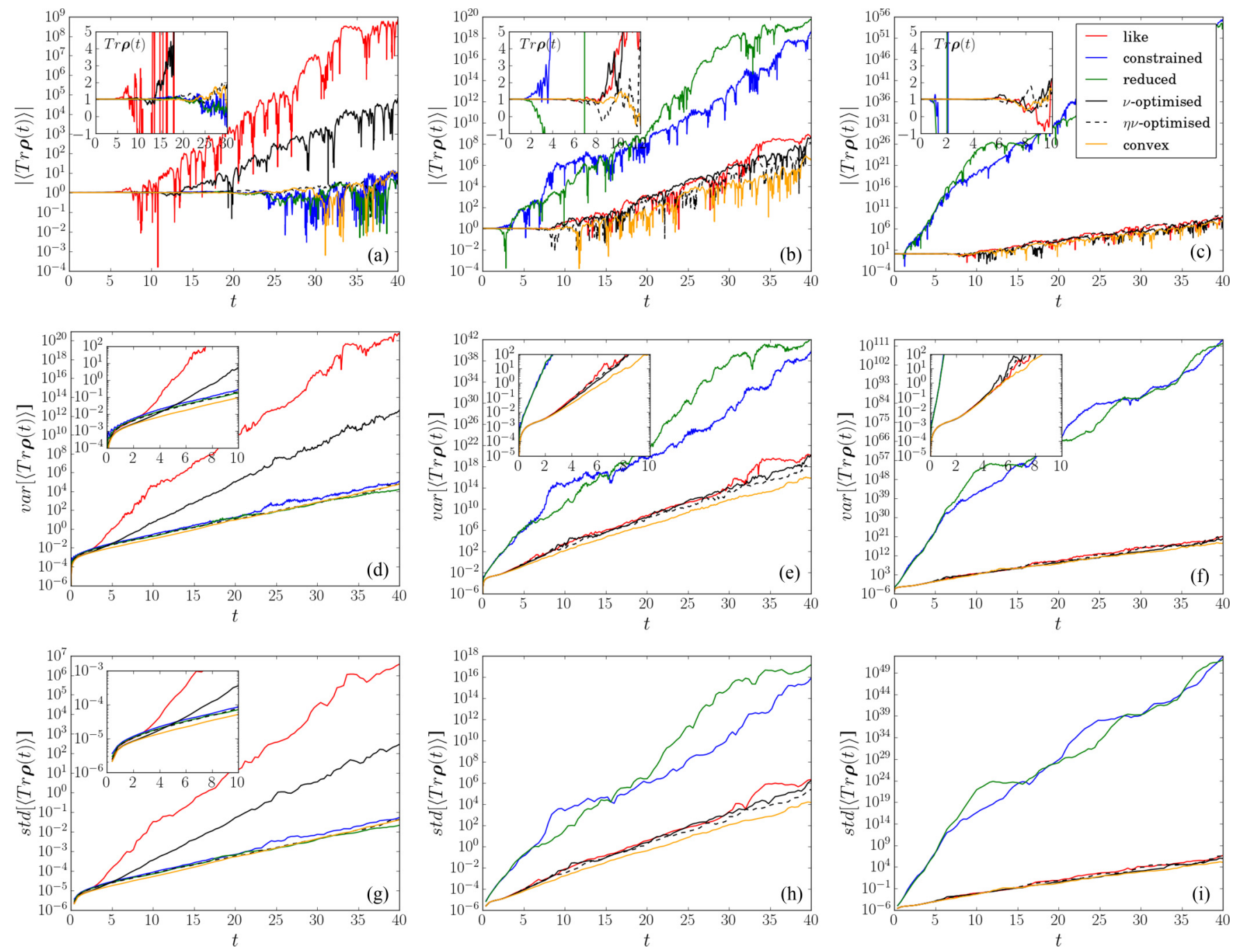

FIG. 6. (a)-(c) The mean value of the trace, $\langle\operatorname{Tr}(\rho(t))\rangle$, calculated using different schemes with the system having been initialized in the state $|1\rangle$ for a constant Hamiltonian. The absolute value of $\langle\operatorname{Tr}(\rho(t))\rangle$ is shown so that the linear growth on a logarithmic scale is clear. The insets highlight the timescales on which the simulation is numerically stable, showing $\langle\operatorname{Tr}(\rho(t))\rangle$ directly; schemes are not shown for timescales beyond which the trace is clearly diverging. (d-f) The variance of the trace and (g)-(i) the standard error of the mean trace calculated over time windows which were 100 time steps long. For columns read from left to right, the inverse temperature increases as $\beta=0.1,1,10$, respectively. The like (red), constrained (blue), and reduced (green) schemes are shown, as well as the $\nu$-optimized scheme which minimizes $\left\langle|v(t)|^{2}\right\rangle$ (black solid), $\eta v$-optimized scheme which minimizes the sum $\left\langle|\eta(t)|^{2}\right\rangle+\left\langle|v(t)|^{2}\right\rangle$ (black dashed), and the application of the convex optimization scheme [21] as implemented using Eqs. (37)-(42) (yellow). All calculations have been done using the same system as in Fig. 3(a). $\Delta=1, \epsilon=-1, \alpha=0.05, \Delta t=10^{-2}, \omega_{c}=25$, and $10^{5}$ realizations. No rescaling of the noises was employed.

In general, either of the optimized schemes represent a very significant improvement in the convergence properties and stability of the trace for the inverse temperatures used, with the growth in the variance of the trace being drastically reduced [Figs. 6(d)-6(f)], allowing an increase in the duration of the stable region [Figs. 6(a)-6(c)]. However, minimizing the typical magnitude of $v$ only is found not sufficient to guarantee this reduction in the variance of the trace for all temperatures, with the performance of the $v$-optimized scheme similar only to the $\eta v$-optimized and convex optimized schemes at lower temperatures $(\beta=1,10)$, but performing much worse at high temperatures $(\beta=0.1)$.

This is understood by comparing Figs. 6(d) and 6(f) for the variance, where the $v$-optimized scheme and the like scheme both fail for small $\beta$ while the $\eta \nu$-optimized scheme performs well. This is caused by the presence of $\operatorname{coth}\left(\frac{1}{2} \beta \hbar \omega\right)$ in $K_{\eta \eta}$ [Eq. (4)], which diverges as $\beta$ becomes small. Since correlation of $v$ with $\eta$ enters via the autocorrelative part of $\eta$ in the reduced scheme, the amplitude of $\eta$ when generated by the reduced scheme will be smaller than when generated by the like scheme, as no other noise component is added to the autocorrelative part. This also explains why the reduced and constrained schemes perform well for $\beta=0.1$ Figs. 6(a), $6(\mathrm{~d})$, and $6(\mathrm{~g})]$. By accounting for this, the $\eta \nu$-optimized scheme is an improvement on the v-optimized scheme despite the fact that $v$ alone is responsible for the intrinsic exponential growth of the trace; this acts as a reminder that these optimization schemes are indirect, in the sense that they do not optimize the properties of the dynamics of the trace directly. 

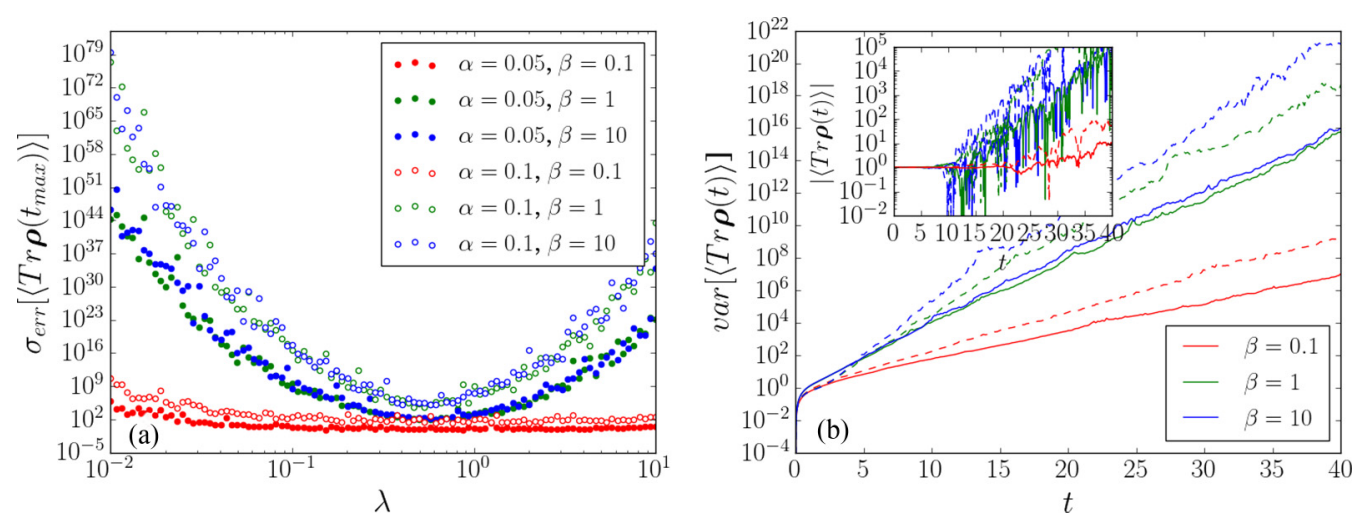

FIG. 7. (a) The standard error of $\langle\operatorname{Tr} \rho(t)\rangle$ at its final time step $t_{\max }$ as a function of the scaling factor $\lambda$ for several values of inverse temperature $\beta$ and coupling strength $\alpha$. For each scaling factor, 1000 runs for real time dynamics were performed. $t_{\max }=40, d t=10^{-3}$, $\omega_{c}=25$, and $\Delta=1, \epsilon=-1$ for $\alpha=0.05,0.1$ and $\beta=0.1,1,10$. In this case, the optimum value of $\lambda$ which minimizes the growth of the trace is $\approx 0.5$. (b) The variance of the trace having used the $\eta v$-optimized scheme with scaling, with a desired ratio between $f_{2}(t)$ and $g_{2}(t)$ of $\lambda=0.5$ (solid lines) for $\beta=0.1,1,10$, with the mean trace shown in the inset. The convex optimized scheme (dashed lines) has been reproduced here from Figs. 6(d)-6(f) for comparison.

Accounting for this temperature dependence, the raw $\eta \nu$ optimized scheme (without any rescaling) and the application of convex optimization are comparable, with the benefit that these schemes are universal rather than depending strongly on the temperature. It is quite fortunate, as if this were not the case, an investigation of this kind would have to be performed for every system when selecting a scheme.

\section{C. $\eta v$-Optimized scheme with rescaling}

In Fig. 7 we apply dynamical scaling to the $f_{2}$ and $g_{2}$ components of $\eta$ and $v$ as generated by the $\eta v$-optimized scheme, Eqs. (33)-(35), with $\zeta=1 / 2$. By comparing in Fig. 7(a) the value of $|\langle\operatorname{Tr} \rho(t)\rangle|$ at the end of a constant Hamiltonian simulation for a range of rescaling values $\lambda \in(0.01,10)$ using the procedure of Sec. III A 6, we find that the optimal value of the scaling is $\lambda=0.5$, which we note is the same value obtained previously for the like scheme [18]. Rescaling the noises with this optimal $\lambda$ using the same parameters as in Fig. 6, we find that the variance of the trace is reduced further, shown in Fig. 7(b) alongside the convex optimized data from Fig. 6 for comparison.

We find that the rescaled $\eta v$-optimized scheme is the best scheme for generating noises which minimize the spread and growth (see inset) of the trace for all the schemes considered, at both high and low temperatures. From a practical perspective, the optimal $\lambda$ can be quickly obtained with only 100 realizations or fewer for each value of $\lambda$, so does not represent a meaningful increase in computational effort.

\section{DISCUSSION AND CONCLUSIONS}

In this paper we have developed a number of competing noise generation schemes, capable of generating complex colored noises appropriate for the implementation of the stochastic Liouville--von Neumann equation. These noises represent the interaction between the system of interest and its environment and must satisfy the correlation functions of Eqs. (4) and (5), with the physical interpretation that averaging over the manifestations of these noises is equivalent to averaging over all possible behaviors of the bath. All of the schemes proposed here do satisfy the desired correlations, but do not otherwise perform equally; that is, the required sample size for convergence is not uniform between schemes, and nor is the quality of the subsequent driven dynamics of the reduced system density matrix. This leads to the important point that there is significant flexibility in the definitions of the noises, as they are not uniquely defined by the correlation functions which they must satisfy.

At all stages in this work, great care has been taken to be as transparent and explicit in the development as possible, in terms of both the presentation of analytical solutions and the numerical implementation of the schemes subsequently developed.

Within the general linear filtering ansatz [Eqs. (14) and (15)] we have identified a subclass of schemes, which we refer to as orthogonal decompositions [17], where the noises are decomposed into components which are correlated with only one other component (or with themselves) and have the beneficial property that zero self-correlation can be fulfilled by construction. There is no limit to the possible choices of the filters with which these components might be generated from white noise, though we focus on two such choices for the cross-correlative components between the $\eta$ and $v$ noises: the delta scheme (Sec. III A 1) where one of the noise components is chosen to be purely white noise, and the like scheme (Sec. III A 3) where the filters are chosen so that one is equal to the other with $\omega \rightarrow-\omega$. The delta scheme represents the worst of the choices, requiring sample sizes of at least $\sim 10^{6}$ for the correlation functions to converge while still producing unstable dynamics for which the trace rapidly diverges to $\pm \infty$. This is a prime demonstration that satisfying the correlation functions alone is not sufficient to guarantee well-behaved dynamics, or that unrealistically large samples might be required before the dynamics converges.

Building on an alternative structure for the noises which cannot be written as an orthogonal decomposition, we followed the arguments in Ref. [20] to develop a scheme which 
chooses either the like or constrained scheme (of Sec.s III A 3 and III A 2, respectively) at each $\omega$ to reduce the average magnitude of the $v$ noise which controls the spreading of the trace of the reduced system density matrix. Crucially, by introducing a mixing function $\tilde{A}(\omega)$ to blend the schemes and performing a minimization in Fourier space to choose it, we were able to ensure that the mixing function was introduced to the filters in Eqs. (27)-(29) correctly such that the properties of the Fourier transforms of the correlation functions were maintained. Further, by exploiting these properties and deriving the Fourier transforms in full, we were able to identify that the enforcement of causality in the $\eta-v$ correlation was responsible for a logarithmic divergence in its Fourier transform. This in turn causes an amplification of the noise power for frequencies around the cutoff frequency of the spectral density of the bath, resulting in weaker convergence than if causality was not required. Fortunately, by employing the Wiener filter for deconvolutions in Sec. III C, we were able to parametrize a weakening of causality in cases where division by zero (or very small numbers) in Fourier space would cause the spectral densities of the noises to diverge, ensuring that any deviation from the theory was well controlled while significantly reducing the $v$ noise power.

Going one step further, we explicitly minimized the average amplitude of both the $v$ noise, and the combined amplitudes of the $\eta$ and $v$ noises together, in the $v$-optimized and $\eta v$-optimized schemes, respectively. We then exploited an additional freedom in the relative amplitudes of correlated noise components by increasing the noise power of one component while reducing the noise power of the other by the same amount so that the correlation functions are unchanged. We showed that analytic minimization of the amplitudes of the noises yields a trivial rescaling, but that direct numerical minimization of the standard error of the trace allows us to obtain an optimal scaling. We emphasize that this scaling is an entirely independent freedom to the mixing function, and suggest that there may be many other freedoms and equivalent noise constructions, leaving space for future work.

Finally, we measured the performance of the aforementioned schemes along with an alternative optimized scheme (Sec. III B) based on convex optimization [21] for a range of inverse temperatures, paying special attention to the properties of the reduced system trace as a measure of the deviation from the physical dynamics, as well as its convergence over a set of realizations. By measuring the variance and standard error of the mean of the trace, and inspecting how the time at which numerical breakdown occurs varies for each scheme, we were able to explain why some schemes performed better at different temperatures than others in terms of competing noise amplitudes between $\eta$ and $\nu$, and clearly identified that the rescaled $\eta \nu$-optimized scheme performed universally the best out of all the schemes at all temperatures. Remarkably, this optimization reduced the variance of the trace by as much as $\sim 10^{95}$ at low temperatures and $\sim 10^{15}$ at high temperatures. The SLN equation is then compared with the exact solution of a simple quantum nondemolition model, for which near perfect agreement is obtained with statistical convergence extending beyond initial dynamics and into the equilibrium regime.
While comparison with other methods was not within the scope of this paper, we remark that methods which use approximate forms of the bath response function and do not rely so heavily on noises, e.g., the hierarchical equations of motions [5,8], achieve well-converged results for strong coupling. However, for weaker coupling or arbitrary spectral densities and bath response functions the SLN remains exact, opening an avenue of research for non-Markovian reservoir engineering [50,51]. We hope that this study will stimulate further work in improving the optimization of the simulation schemes and consequently will open avenues for practical numerical simulations of open quantum systems using SLN and ESLN approaches.

\section{ACKNOWLEDGMENTS}

The first two authors contributed equally to this work. D.M. and M.A.L. are supported by the EPSRC Centre for Doctoral Training in Cross-Disciplinary Approaches to NonEquilibrium Systems (CANES, Grant No. EP/L015854/1). Calculations in this paper were performed using the King' $\mathrm{s}$ College HPC cluster Gravity.

\section{APPENDIX A: FOURIER TRANSFORMS}

In deriving the different noise generation schemes (Sec. III), it was necessary to use the properties of the Fourier transform of the $\eta-\eta$ correlation function $K_{\eta \eta}(t)$ and the $\eta-v$ correlation function $K_{\eta v}(t)$ which we reproduce here.

$$
\text { 1. } \tilde{K}_{\eta \eta}(\omega)
$$

Recalling the definition of $K_{\eta \eta}(t)$ [Eq. (4)], its Fourier transform $\tilde{K}_{\eta \eta}(\omega)$ is

$$
\tilde{K}_{\eta \eta}(\omega)=\frac{\hbar}{2} \int_{-\infty}^{\infty} d t \int_{0}^{\infty} \frac{d \omega^{\prime}}{\pi} \Lambda\left(\omega^{\prime}\right)\left[e^{-i\left(\omega-\omega^{\prime}\right) t}+e^{-i\left(\omega+\omega^{\prime}\right) t}\right]
$$

where we have used the shorthand $\Lambda(\omega)=J(\omega) \operatorname{coth}\left(\frac{1}{2} \beta \hbar \omega\right)$ and replaced the cosine with complex exponentials. Using the definition of the $\delta$ function to remove the time integral,

$$
\delta(\omega)=\frac{1}{2 \pi} \int_{-\infty}^{\infty} d t e^{ \pm i \omega t}
$$

we arrive at the final result,

$$
\begin{aligned}
\tilde{K}_{\eta \eta}(\omega) & =\hbar \int_{0}^{\infty} d \omega^{\prime} \Lambda\left(\omega^{\prime}\right)\left[\delta\left(\omega-\omega^{\prime}\right)+\delta\left(\omega+\omega^{\prime}\right)\right] \\
& =\hbar \Lambda(|\omega|)
\end{aligned}
$$

and we can see that $\tilde{K}_{\eta \eta}(\omega)$ is both real, even and everywhere positive.

$$
\text { 2. } \tilde{K}_{\eta v}(\omega)
$$

Recalling the definition of $K_{\eta v}(t)$, Eq. (5), its Fourier transform $\tilde{K}_{\eta v}(\omega)$ is

$$
\begin{aligned}
\tilde{K}_{\eta \nu}(\omega)= & \frac{1}{2 i \pi} \int_{0}^{\infty} \frac{d \omega^{\prime}}{\pi} J\left(\omega^{\prime}\right) \lim _{\epsilon \rightarrow 0^{+}} \int_{-\infty}^{\infty} \frac{d \Omega}{\Omega+i \epsilon} \\
& \times \int_{-\infty}^{\infty} d t\left[e^{-i\left(\omega+\Omega-\omega^{\prime}\right) t}-e^{-i\left(\omega+\Omega+\omega^{\prime}\right) t}\right],
\end{aligned}
$$


where we have replaced the Heaviside step function with

$$
\Theta(t)=\lim _{\epsilon \rightarrow 0^{+}}-\frac{1}{2 i \pi} \int_{-\infty}^{\infty} d \Omega \frac{e^{-i \Omega t}}{\Omega+i \epsilon}
$$

and replaced the sine with complex exponentials. Again, recognizing the definition of the $\delta$ function, Eq. (A2), to remove the time integral and then using the $\delta$ functions to evaluate the $\Omega$ integral, we arrive at the following:

$$
\begin{aligned}
& \tilde{K}_{\eta \nu}(\omega) \\
& \quad=-i \lim _{\epsilon \rightarrow 0^{+}} \int_{0}^{\infty} \frac{d \omega^{\prime}}{\pi} J\left(\omega^{\prime}\right)\left[\frac{1}{\omega^{\prime}-\omega+i \epsilon}+\frac{1}{\omega^{\prime}+\omega-i \epsilon}\right] .
\end{aligned}
$$

We then take the $\epsilon \rightarrow 0^{+}$limit to remove the pole,

$$
\lim _{\epsilon \rightarrow 0^{+}} \frac{1}{\omega^{\prime}-\omega \pm i \epsilon}=\mathcal{P} \frac{1}{\omega^{\prime}-\omega} \mp i \pi \delta\left(\omega^{\prime}-\omega\right)
$$

( $\mathcal{P}$ stands for Cauchy principal value), to obtain

$$
\begin{aligned}
\tilde{K}_{\eta \nu}(\omega)= & \int_{0}^{\infty} d \omega^{\prime} J\left(\omega^{\prime}\right)\left[\delta\left(\omega^{\prime}+\omega\right)-\delta\left(\omega^{\prime}-\omega\right)\right] \\
& -\frac{i}{\pi} f_{0}^{\infty} d \omega^{\prime} J\left(\omega^{\prime}\right)\left(\frac{1}{\omega^{\prime}-\omega}+\frac{1}{\omega^{\prime}+\omega}\right) \\
= & -\operatorname{sgn}(\omega) J(|\omega|)-\frac{2 i}{\pi} f_{0}^{\infty} d \omega^{\prime} \frac{\omega^{\prime} J\left(\omega^{\prime}\right)}{\omega^{\prime 2}-\omega^{2}},
\end{aligned}
$$

where $f$ also corresponds to Cauchy principal value. Note that $\tilde{R}(\omega)=-i \tilde{K}_{\eta \nu}(\omega)$, so we immediately see that the real part of $\tilde{K}_{\eta v}$ (and the imaginary part of $\tilde{R}$ ) is odd.

\section{The singularity in $\tilde{K}_{\eta v}(\omega)$}

From Eq. (A9), we see that the imaginary part of $\tilde{K}_{\eta \nu}(\omega)$ has an instability at $\omega^{\prime}=\omega$ that is integrable due to the Cauchy principle value. By writing $\operatorname{Im}\left[\tilde{K}_{\eta \nu}(\omega)\right]$ as

$$
\operatorname{Im}\left[\tilde{K}_{\eta \nu}(\omega)\right]=-\frac{2}{\pi} f_{0}^{\omega_{c}} d \omega^{\prime} \frac{\omega^{\prime 2} f\left(\omega^{\prime}\right)}{\omega^{\prime 2}-\omega^{2}},
$$

where $f(\omega)=\left[1+\left(\frac{\omega}{\omega_{c}}\right)^{2}\right]^{-2}$ and we have used the fact that $J(\omega)$ is zero outside of the range of $\omega$ values $0 \leqslant \omega \leqslant \omega_{c}$, we integrate it as follows:

$$
\begin{aligned}
\int_{0}^{\omega_{c}} d x \frac{x^{2} f(x)}{x^{2}-\omega^{2}}= & \int_{0}^{\omega_{c}} d x \frac{x^{2}[f(x)-f(\omega)]}{x^{2}-\omega^{2}} \\
& +f(\omega) f_{0}^{\omega_{c}} d x \frac{x^{2}}{x^{2}-\omega^{2}} .
\end{aligned}
$$

Only the second term contains the instability, which can be handled as

$$
\begin{aligned}
f_{0}^{\omega_{c}} d x \frac{x^{2}}{x^{2}-\omega^{2}} & =\int_{0}^{\omega_{c}} d x+\omega^{2} f_{0}^{\omega_{c}} \frac{d x}{x^{2}-\omega^{2}} \\
& =\omega_{c}+\frac{\omega}{2} \ln \left|\frac{\omega_{c}-\omega}{\omega_{c}+\omega}\right|
\end{aligned}
$$

by breaking the Cauchy principal value integral into an integral from 0 to $\omega-\epsilon$ and from $\omega+\epsilon$ to $\omega_{c}$ and seeing that the result is independent of the infinitesimal $\epsilon$. Hence Eq. (A11) converges in the Cauchy sense, though a logarithmic divergence at $\omega= \pm \omega_{c}$ has appeared.
Applying this argument to $\operatorname{Im}\left[\tilde{K}_{\eta \nu}(\omega)\right]$ and simplifying, we arrive at

$$
\begin{aligned}
\operatorname{Im}\left[\tilde{K}_{\eta \nu}(\omega)\right]= & -\frac{2}{\pi}\left(\omega_{c}+\frac{\omega}{2} \ln \left|\frac{\omega_{c}-\omega}{\omega_{c}+\omega}\right|\right) f(\omega) \\
& +\frac{2}{\pi} \frac{\omega_{c}^{3}}{\omega_{c}^{2}+\omega^{2}} \int_{0}^{1} d x \frac{x^{2}}{\left(1+x^{2}\right)^{2}} \\
& \times\left[\omega_{c}^{2} x^{2}+2 \omega_{c}^{2}+\omega^{2}\right] .
\end{aligned}
$$

The remaining integrals are then evaluated by relation to the arctangent to give

$$
\begin{aligned}
\operatorname{Im}\left[\tilde{K}_{\eta \nu}(\omega)\right]= & -\frac{2}{\pi}\left(\omega_{c}+\frac{\omega}{2} \ln \left|\frac{\omega_{c}-\omega}{\omega_{c}+\omega}\right|\right)\left[1+\left(\frac{\omega}{\omega_{c}}\right)^{2}\right]^{-2} \\
& +\frac{1}{4 \pi} \frac{\omega_{c}^{3}}{\left(\omega_{c}^{2}+\omega^{2}\right)}\left[(6-\pi) \omega_{c}^{2}+(\pi-2) \omega^{2}\right] .
\end{aligned}
$$

Thus the imaginary part of $\tilde{K}_{\eta v}$ is even and the real part of $\tilde{R}$ is odd.

The emergence of the logarithmic divergence when $\omega=$ $\pm \omega_{c}$ originates with the presence of the Heaviside step function in the $\eta v$ correlation of Eq. (5), which by Eq. (A5) and the use of the $\delta$ function introduces the singularity $\sim \frac{1}{\omega^{\prime 2}-\omega^{2}}$ in Eq. (A9). Since the Heaviside function is an intrinsic part of the $\eta v$ correlation, that is, it was rigorously derived [16] rather than being included artificially, its presence is required by the theory such that removing it any way would not be formally correct.

\section{APPENDIX B: OPTIMIZED MIXING FUNCTION $\tilde{A}(\omega)$}

\section{Symmetry of $\tilde{A}(\omega)$}

It is possible to determine some general properties of the real and imaginary parts of the mixing function $\tilde{A}(\omega)=$ $\tilde{A}_{1}(\omega)+i \tilde{A}(\omega)$ simply from the properties of $\tilde{R}$. Recalling Eq. (20) coming from $\tilde{K}_{\eta v}$ and generalizing to arbitrarily many cross-correlative components,

$$
\tilde{f}_{1}(\omega) \tilde{g}_{1}(-\omega)+2 \sum_{j=2} \tilde{f}_{j}(\omega) \tilde{g}_{j}(-\omega)=\tilde{R}(\omega),
$$

we can then make use of the fact that $\left\{f_{j}(t)\right\}$ and $\left\{g_{j}(t)\right\}$ are all real functions. Thus their Fourier transforms must have even real parts and odd imaginary parts, since $\tilde{f}^{*}(\omega)=\tilde{f}(-\omega)$ for any real function $f(t)$. Then, from Eqs. (A9) and (A14), we see that $\tilde{R}(\omega)=\tilde{R}_{1}(\omega)+i \tilde{R}_{2}(\omega)$ has even real part $\tilde{R}_{1}$ and odd imaginary part $\tilde{R}_{2}$. Using the shorthand $\tilde{f}_{j}=\tilde{f}_{j}^{R}+i \tilde{f}_{j}^{I}$ and $\tilde{g_{j}}={\tilde{g_{j}}}^{R}+i{\tilde{g_{j}}}^{I}$ for the real and imaginary parts of the filters, we thus have

$$
\begin{aligned}
\tilde{R}_{1}(\omega)= & \sqrt{\tilde{K}_{\eta \eta}(\omega)} \tilde{g}_{1}^{R}(\omega) \\
& +2 \sum_{j=2}\left[\tilde{f}_{j}^{R}(\omega) \tilde{g}_{j}^{R}(\omega)+\tilde{f}_{1}^{I}(\omega) \tilde{g}_{j}^{I}(\omega)\right],
\end{aligned}
$$

for the real part, where we have used the fact that the real parts of the filters are even and that the imaginary parts are odd, and that $\tilde{f}_{1}(\omega)=\sqrt{\tilde{K}_{\eta \eta}(\omega)}$ is real. Similarly for the imaginary part 
we have

$$
\begin{aligned}
\tilde{R}_{2}(\omega)= & \sqrt{\tilde{K}_{\eta \eta}(\omega)} \tilde{g}_{1}^{I}(\omega) \\
& +2 \sum_{j=2}\left[\tilde{f}_{j}^{R}(\omega) \tilde{g}_{j}^{I}(\omega)-\tilde{f}_{j}^{I}(\omega) \tilde{g}_{j}^{R}(\omega)\right] .
\end{aligned}
$$

For the case we are considering where we include only the $j=2$ term, and using the filters given by Eqs. (26)-(29), we can determine the symmetry properties of the real and imaginary parts of the mixing function $\tilde{A}(\omega)=\tilde{A}_{1}(\omega)+i \tilde{A}_{2}(\omega)$. Since $\tilde{g}_{1}(-\omega)=\tilde{g}_{1}^{*}(\omega)$, the general $\tilde{g}_{1}$ filter $(28)$ requires

$$
\begin{aligned}
& {\left[1-\tilde{A}_{1}(\omega)-i \tilde{A}_{2}(\omega)\right]\left[\tilde{R}_{1}(\omega)+i \tilde{R}_{2}(\omega)\right]} \\
& \quad=\left[1-\tilde{A}_{1}(-\omega)+i \tilde{A}_{2}(-\omega)\right]\left[\tilde{R}_{1}(-\omega)-i \tilde{R}_{2}(-\omega)\right],
\end{aligned}
$$

which constrains the real and imaginary parts as

$$
\begin{gathered}
{\left[\tilde{A}_{1}(\omega)-\tilde{A}_{1}(-\omega)\right] \tilde{R}_{1}(\omega)=\left[\tilde{A}_{2}(\omega)+\tilde{A}_{2}(-\omega)\right] \tilde{R}_{2}(\omega), \quad(\mathrm{B})} \\
-\left[\tilde{A}_{1}(\omega)-\tilde{A}_{1}(-\omega)\right] \tilde{R}_{2}(\omega)=\left[\tilde{A}_{2}(\omega)+\tilde{A}_{2}(-\omega)\right] \tilde{R}_{1}(\omega),
\end{gathered}
$$

respectively, where we have again used the symmetry properties of $\tilde{R}$. Assuming that $\tilde{A}_{1}(\omega)-\tilde{A}_{1}(-\omega) \neq 0$, then $\tilde{A}_{2}(\omega)+$ $\tilde{A}_{2}(-\omega) \neq 0$ and dividing Eq. (B5) by (B6) would require that $\tilde{R}_{1}(\omega)^{2}=-\tilde{R}_{2}(\omega)^{2}$ which is obviously incorrect since they are both real. Therefore $\tilde{A}_{1}(\omega)=\tilde{A}_{1}(-\omega)$ and then $\tilde{A}_{2}(\omega)=$ $-\tilde{A}_{2}(-\omega)$, i.e., the real part of the mixing function must be even and the imaginary part must be odd.

Note that the same analysis of Eqs. (27) and (29) results in exactly the same conditions for the mixing function.

\section{Minimizing magnitude of $v(t)$}

Starting with $v$ as it is written in Eq. (17), its magnitude is

$\left\langle|v(t)|^{2}\right\rangle=\int \frac{d \omega}{2 \pi} \tilde{K}_{\nu v^{*}}(\omega)=\int \frac{d \omega}{2 \pi}\left\{2\left|\tilde{g}_{1}(\omega)\right|^{2}+2\left|\tilde{g}_{2}(\omega)\right|^{2}\right\}$,

where $K_{v v^{*}}(t)=\left\langle v(t) v^{*}(t)\right\rangle$ and we have made use of Parseval's theorem to remove the exponential factor associated with the inverse Fourier transform. While it may at first seem strange that there is no time dependence on the right-hand side, there is no reason why $\langle|v(t)|\rangle^{2}$ should not be stationary. In fact, this apparent stationarity is a direct consequence of the form of the noises (16) and (17) containing time differences in the filters. Substituting in the above expression Eqs. (28) and (29) and making use of the fact that $\tilde{K}_{\eta \eta}(\omega)$ is real and even, that the real part of $\tilde{R}(\omega)=\tilde{R}_{1}(\omega)+i \tilde{R}_{2}(\omega)$ is even while the imaginary part is odd, we obtain Eq. (30) for $\left\langle|v(t)|^{2}\right\rangle$. We have also used the fact that the magnitude of a complex function whose real and imaginary parts are either even or odd is always real, even and positive. The aim now is to minimize $\tilde{K}_{v v^{*}}(\omega)$ with respect to the real and imaginary parts of the mixing function $\tilde{A}(\omega)=\tilde{A}_{1}(\omega)+i \tilde{A}_{2}(\omega)$ at each $\omega$ value, where we know that the real part of $\tilde{A}$ should be even and the imaginary part odd. Starting with the real part,

$$
\frac{d \tilde{K}_{\nu v^{*}}(\omega)}{d \tilde{A}_{1}(\omega)}=\frac{|\tilde{R}(\omega)|}{\tilde{K}_{\eta \eta}(\omega)}\left[4|\tilde{R}(\omega)|+\tilde{K}_{\eta \eta}(\omega) \frac{\tilde{A}_{1}(\omega)}{|\tilde{A}(\omega)|}\right]=0,
$$

which yields the following constraint on $\tilde{A}_{1}$ and its magnitude:

$$
\tilde{A}_{1}(\omega)\left[4|\tilde{R}(\omega)|+\frac{\tilde{K}_{\eta \eta}(\omega)}{|\tilde{A}(\omega)|}\right]=4|\tilde{R}(\omega)| .
$$

Minimizing with respect to the imaginary part of the mixing function then gives

$$
\frac{d \tilde{K}_{\nu v^{*}}(\omega)}{d \tilde{A}_{2}(-\omega)}=\frac{|\tilde{R}(\omega)|}{\tilde{K}_{\eta \eta}(\omega)} \tilde{A}_{2}(-\omega)\left[4|\tilde{R}(\omega)|+\frac{\tilde{K}_{\eta \eta}(\omega)}{|\tilde{A}(-\omega)|}\right]=0,
$$

so that $\tilde{A}$ must either be real with $\tilde{A}_{2}(\omega)=0$, or the terms within the square brackets must equal zero. If the latter was true, then Eq. (B9) would require that $|\tilde{R}(\omega)|=0$ which is certainly not correct (also, both terms inside the square brackets are positive), so $\tilde{A}$ is indeed real, $\tilde{A}(\omega)=\tilde{A}_{1}(\omega)$. Equation (B9) then gives

$$
\tilde{A}(\omega)=1-\operatorname{sgn}[\tilde{A}(\omega)] \frac{\tilde{K}_{\eta \eta}(\omega)}{4|\tilde{R}(\omega)|} .
$$

Since $\tilde{A}<0$ would lead to a contradiction $\left(\tilde{K}_{\eta \eta} /|\tilde{R}|\right.$ is always positive, so the right-hand side would then be positive), we must conclude that $\tilde{A}$ is a positive function, leading finally to

$$
\tilde{A}(\omega)=1-\frac{\tilde{K}_{\eta \eta}(\omega)}{4|\tilde{R}(\omega)|} .
$$

Substituting this $\tilde{A}$ into the filters of Eqs. (27)-(29) gives Eqs. (33)-(35). It is worth noting that the division by $|\tilde{R}(\omega)|$ does not require serious consideration using the deconvolution procedure as detailed in Sec. (IIIC) since it appears as only a combination $\tilde{R}(\omega) /|\tilde{R}(\omega)|$. Since $|\tilde{R}| \geqslant \tilde{R}$, this ratio will always converge without any division by small numbers.

\section{Minimizing magnitudes of $\eta(t)$ and $v(t)$}

We now consider the sum

$$
\begin{aligned}
\left\langle|\eta(t)|^{2}\right\rangle+\left\langle|v(t)|^{2}\right\rangle= & \int \frac{d \omega}{2 \pi}\left[\tilde{K}_{\eta \eta}(\omega)+\tilde{K}_{v v^{*}}(\omega)\right] \\
= & \int \frac{d \omega}{2 \pi}\left[\tilde{f}_{1}(\omega)^{2}+2\left|\tilde{f}_{2}(\omega)\right|^{2}\right. \\
& \left.+2\left|\tilde{g}_{1}(\omega)\right|^{2}+2\left|\tilde{g}_{2}(\omega)\right|^{2}\right],
\end{aligned}
$$

and insert Eqs. (26)-(29) to obtain

$$
\begin{aligned}
\tilde{K}_{\eta \eta}(\omega)+\tilde{K}_{v v^{*}}(\omega)= & \tilde{K}_{\eta \eta}(\omega)+2|\tilde{R}(\omega)||\tilde{A}(\omega)| \\
& +2 \frac{|\tilde{R}(\omega)|^{2}}{\tilde{K}_{\eta \eta}(\omega)}|1-\tilde{A}(\omega)|^{2},
\end{aligned}
$$

where we have again used the properties of $\tilde{K}_{\eta \eta}$ and $\tilde{R}$, and the fact that $\tilde{A}$ must be an even function. The essential difference of the obtained expression from Eq. (30) for $\left\langle|v(t)|^{2}\right\rangle$ is only in the factor of two in the last term. Hence, repeating the analysis of the previous Appendix we obtain

$$
\tilde{A}(\omega)=1-\frac{\tilde{K}_{\eta \eta}(\omega)}{2|\tilde{R}(\omega)|} .
$$

Note that the same result can be obtained without initially using the fact that $\tilde{A}$ is an even function, in which case it is more convenient to write $\tilde{A}$ in the form $\tilde{A}(\omega)=r(\omega) e^{i \theta(\omega)}$. 
[1] P. W. Shor, Scheme for reducing decoherence in quantum computer memory, Phys. Rev. A 52, R2493(R) (1995).

[2] U. Weiss, Quantum Dissipative Systems (World Scientific, Singapore, 2012), Vol. 13.

[3] R. P. Feynman and F. L. Vernon Jr., The theory of a general quantum system interacting with a linear dissipative system, Ann. Phys. 281, 547 (2000).

[4] J. Shao, Decoupling quantum dissipation interaction via stochastic fields, Chem. Phys. 120, 5053 (2004).

[5] Y. Yan, F. Yang, Y. Liu, and J. Shao, Hierarchical approach based on stochastic decoupling to dissipative systems, Chem. Phys. Lett. 395, 216 (2004).

[6] Y. A. Yan and J. Shao, Stochastic description of quantum Brownian dynamics, Front. Phys. 11, 110309 (2016).

[7] Y. Zhou, Y. Yan, and J. Shao, Stochastic simulation of quantum dissipative dynamics, Europhys. Lett. 72, 334 (2005).

[8] Y. Zhou and J. Shao, Solving the spin-boson model of strong dissipation with flexible random-deterministic scheme, J. Chem. Phys. 128, 034106 (2008).

[9] D. Suess, A. Eisfeld, and W. T. Strunz, Hierarchy of Stochastic Pure States for Open Quantum System Dynamics, Phys. Rev. Lett. 113, 150403 (2014).

[10] P. P. Orth, A. Imambekov, and K. Le Hur, Nonperturbative stochastic method for driven spin-boson model, Phys. Rev. B 87, 014305 (2013).

[11] N. Makri and D. E. Makarov, Tensor propagator for iterative quantum time evolution of reduced density matrices. I. Theory, J. Chem. Phys. 102, 4600 (1995).

[12] L. Diósi, N. Gisin, and W. T. Strunz, Non-Markovian quantum state diffusion, Phys. Rev. A 58, 1699 (1998).

[13] J. T. Stockburger and H. Grabert, Non-Markovian quantum state diffusion, Chem. Phys. 268, 249 (2001).

[14] J. T. Stockburger and H. Grabert, Exact $c$-Number Representation of Non-Markovian Quantum Dissipation, Phys. Rev. Lett. 88, 170407 (2002).

[15] J. T. Stockburger, Simulating spin-boson dynamics with stochastic Liouville-von Neumann equations, Chem. Phys. 296, 159 (2004).

[16] G. M. G. McCaul, C. D. Lorenz, and L. Kantorovich, Partition-free approach to open quantum systems in harmonic environments: An exact stochastic Liouville equation, Phys. Rev. B 95, 125124 (2017).

[17] G. M. G. McCaul, C. D. Lorenz, and L. Kantorovich, Driving spin-boson models from equilibrium using exact quantum dynamics, Phys. Rev. B 97, 224310 (2018).

[18] M. A. Lane, D. Matos, I. J. Ford, and L. Kantorovich, Exactly thermalized quantum dynamics of the spin-boson model coupled to a dissipative environment, Phys. Rev. B 101, 224306 (2020).

[19] H. Grabert, P. Schramm, and G. Ingold, Quantum Brownian motion: The functional integral approach, Phys. Rep. 168, 115 (1988).

[20] H. Imai, Y. Ohtsuki, and H. Kono, Application of stochastic Liouville-von Neumann equation to electronic energy transfer in FMO complex, Chem. Phys. 446, 134 (2015).

[21] K. Schmitz and J. T. Stockburger, A variance reduction technique for the stochastic Liouville-von Neumann equation, Eur. Phys. J.: Spec. Top. 227, 1929 (2019).
[22] L. Duan and G. Guo, Reducing decoherence in quantumcomputer memory with all quantum bits coupling to the same environment, Phys. Rev. A 57, 737 (1998).

[23] T. A. Costi and R. H. McKenzie, Entanglement between a qubit and the environment in the spin-boson model, Phys. Rev. A 68 , 034301 (2003).

[24] C. H. van der Wal, F. K. Wilhelm, C. Harmans, and J. E. Mooij, Engineering decoherence in Josephson persistentcurrent qubits, Eur. Phys. J. B 31, 111 (2003).

[25] A. Kopp and K. Le Hur, Universal and Measurable Entanglement Entropy in the Spin-Boson Model, Phys. Rev. Lett. 98, 220401 (2007).

[26] W. Cui, Z. Xi, and Y. Pan, Non-Markovian entanglement dynamics between two coupled qubits in the same environment, J. Phys. A: Math. Theor. 42, 155303 (2009).

[27] Y. Makhlin, G. Schön, and A. Shnirman, Quantum-state engineering with Josephson-junction devices, Rev. Mod. Phys. 73, 357 (2001).

[28] J. Liu, L. Fu, B. Y. Ou, S. G. Chen, D. Choi, B. Wu, and Q. Niu, Theory of nonlinear Landau-Zener tunneling, Phys. Rev. A 66, 023404 (2002).

[29] D. Valenti, C. Guarcello, and B. Spagnolo, Switching times in long-overlap Josephson junctions subject to thermal fluctuations and non-Gaussian noise sources, Phys. Rev. B 89, 214510 (2014)

[30] P. P. Orth, D. Roosen, W. Hofstetter, and K. Le Hur, Dynamics, synchronization, and quantum phase transitions of two dissipative spins, Phys. Rev. B 82, 144423 (2010).

[31] P. P. Orth, I. Stanic, and K. Le Hur, Dissipative quantum Ising model in a cold-atom spin-boson mixture, Phys. Rev. A 77, 051601(R) (2008).

[32] D. M. Berns, M. S. Rudner, S. O. Valenzuela, K K. Berggren, W. D. Oliver, L. S. Levitov, and T. P. Orlando, Amplitude spectroscopy of a solid-state artificial atom, Nature (London) 455, 51 (2008).

[33] S. W. Smith, The scientist and engineer's guide to digital signal processing, California Technical Pub., San Diego (1997).

[34] P. C. Hansen, Deconvolution and regularization with Toeplitz matrices, Numer. Algorithms 29, 323 (2002).

[35] A. O. Caldeira and A. J. Leggett, Path integral approach to quantum Brownian motion, Physica A: Stat. Mech. Appl. 121, 587 (1983).

[36] A. V. Oppenheim, Discrete-Time Signal Processing (Upper Saddle River, New Jersey, 1999).

[37] J. Shao, Rigorous representation and exact simulation of real Gaussian stationary processes, Chem. Phys. 375, 378 (2010).

[38] J. L. Starck, E. Pantin, and F. Murtagh, Deconvolution in astronomy: A review, Publ. Astron. Soc. Pac. 114, 1051 (2002).

[39] N. Wiener, Extrapolation, Interpolation and Smoothing of Stationary Time Series with Engineering Applications (MIT Press Cambridge, Massachusetts, 1949).

[40] D. Van de Sompel, L. S. Sasportas, J. V. Jokerst, and S. S. Gambhir, Comparison of deconvolution filters for photoacoustic tomography, PLoS ONE 11, e0152597 (2016).

[41] C. Zener, Non-adiabatic crossing of energy levels, Proc. R. Soc. London A 137, 696 (1932).

[42] C. Wittig, The Landau-Zener formula, J. Phys. Chem. B 109, 8428 (2005). 
[43] A. G. Rojo, Matrix exponential solution of the Landau-Zener problem, arXiv:1004.2914 (2010).

[44] K. Saito, M. Wubs, S. Kohler, Y. Kayanuma, and P. Hänggi, Dissipative Landau-Zener transitions of a qubit: Bath-specific and universal behavior, Phys. Rev. B 75, 214308 (2007).

[45] P. Nalbach and M. Thorwart, Landau-Zener Transitions in a Dissipative Environment: Numerically Exact Results, Phys. Rev. Lett. 103, 220401 (2009).

[46] M. Wubs, K. Saito, S. Kohler, P. Hänggi, and Y. Kayanuma, Gauging a Quantum Heat Bath with Dissipative Landau-Zener Transitions, Phys. Rev. Lett. 97, 200404 (2006).

[47] V. B. Braginsky, Y. I. Vorontsov, and K. S. Thorne, Quantum nondemolition measurements, Science 209, 547 (1980).
[48] A. Lupascu, S. Saito, T. Picot, P. C. De Groot, C. J. P. M. Harmans, and J. E. Mooij, Quantum non-demolition measurement of a superconducting two-level system, Nat. Phys. 3, 119 (2007).

[49] J. Shao, M. Ge, and H. Cheng, Decoherence of quantumnondemolition systems, Phys. Rev. E 53, 1243 (1996).

[50] H. P. Breuer, E. M. Laine, J. Piilo, and B. Vacchini, Colloquium: Non-Markovian dynamics in open quantum systems, Rev. Mod. Phys. 88, 021002 (2016).

[51] J. S. Tang, C. F. Li, Y. L. Li, X. B. Zou, G. C. Guo, H. P. Breuer, E. M. Laine, and J. Piilo, Measuring non-Markovianity of processes with controllable system-environment interaction, Europhys. Lett. 97, 10002 (2012). 